\title{
The Lorenz Attractor, a Paradigm for Chaos
}

\author{
Étienne Ghys
}

\begin{abstract}
It is very unusual for a mathematical or physical idea to disseminate into the society at large. An interesting example is chaos theory, popularized by Lorenz's butterfly effect: "does the flap of a butterfly's wings in Brazil set off a tornado in Texas?" A tiny cause can generate big consequences! Mathematicians (and non mathematicians) have known this fact for a long time! Can one adequately summarize chaos theory is such a simple minded way? In this review paper, I would like first of all to sketch some of the main steps in the historical development of the concept of chaos in dynamical systems, from the mathematical point of view. Then, I would like to present the present status of the Lorenz attractor in the panorama of the theory, as we see it Today.
\end{abstract}

Translation by Stéphane Nonnenmacher from the original French text ${ }^{1}$

\section{Introduction}

The "Lorenz attractor" is the paradigm for chaos, like the French verb "aimer" is the paradigm for the verbs of the 1st type. Learning how to conjugate "aimer" is not sufficient to speak French, but it is doubtlessly a necessary step. Similarly, the close observation of the Lorenz attractor does not suffice to understand all the mechanisms of deterministic chaos, but it is an unavoidable task for this aim. This task is also quite pleasant, since this object is beautiful, both from the mathematical and aesthetic points of view. It is not surprising that the "butterfly effect" is one of the few mathematical concepts widely known among non-scientists.

In epistemology, a paradigm is "a dominant theoretical concept, at a certain time, in a given scientific community, on which a certain scientific domain bases the questions to be asked, and the explanations to be given." ${ }^{2}$ The Lorenz attractor has indeed played this role in the modern theory of dynamical systems, as I will try

\footnotetext{
${ }^{1}$ http://www.bourbaphy.fr/ghys.pdf

${ }^{2}$ Trésor de la Langue Française.
} 


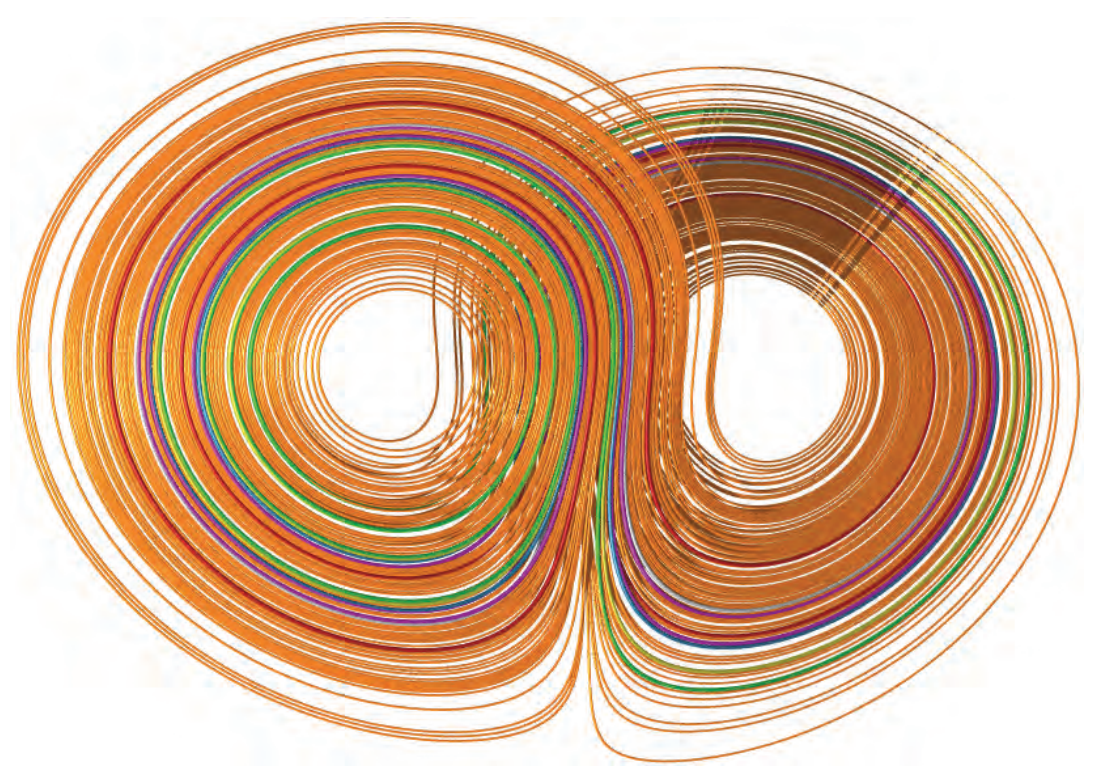

FiguRE 1. The Lorenz attractor

to explain. The Lorenz dynamics features an ensemble of qualitative phenomena which are thought, today, to be present in "generic" dynamics.

According to the spirit of this seminar, this text is not written exclusively for mathematicians. The article [81] is another accessible reference for a description of the Lorenz attractor. The nice book "Dynamics beyond uniform hyperbolicity. A global geometric and probabilistic perspective" by Bonatti, Díaz and Viana gives an account of the state of the art on the subject, but is aimed at experts [16].

In a first step, I wish to rapidly present two past paradigms which have been superseded by the Lorenz attractor: the "(quasi-)periodic dynamics" and the "hyperbolic dynamics". Lorenz's article dates back to 1963, but it was really noticed by mathematicians only a decade later, and it took another decade to realize the importance of this example. One could regret this lack of communication between mathematicians and physicists, but this time was also needed for the hyperbolic paradigm to consolidate, before yielding to its nonhyperbolic successors ${ }^{3}$. In a second step I will present the Lorenz butterfly as it is understood today, focussing on the topological and statistical aspects. Then, I will try to sketch the general picture of dynamical systems, in the light of an ensemble of (optimistic) conjectures due to Palis.

Chaos theory is often described from a negative viewpoint: the high sensitivity to initial conditions makes it impossible to practically determine the future evolution of a system, because these initial conditions are never known with total

${ }^{3}$ For a historical presentation of chaos theory, see for instance [8]. 
precision. Yet, the theory would be rather poor if it was limited to this absence of determinism and did not encompass any deductive aspect. On the contrary, I want to insist on the fact that, by asking the good questions, the theory is able to provide rich and nontrivial information, and leads to a real understanding of the dynamics.

There remains a lot of work to do, halfway between mathematics and physics, in order to understand whether this "little ordinary differential equation" can account for meteorological phenomena, which initially motivated Lorenz. Long is the way between these differential equations and the "true" Navier-Stokes partial differential equations at the heart of the physical problem. Due to my incompetence, I will not dwell on this important question.

I prefer to consider the butterfly as a nice gift from physicists to mathematicians!

I thank Aurélien Alvarez, Maxime Bourrigan, Pierre Dehornoy, Jos Leys and Michele Triestino for their help when preparing those notes. I also thank Stéphane Nonnenmacher for his excellent translation of the French version of this paper.

\section{Starting with a few quotations}

I would like to start by a few quotations, which illustrate the evolution of the opinions on dynamics across the last two centuries.

Let us start with Laplace's famous definition of determinism, in his 1814 "Essai philosophique sur les probabilités" [41]:

We ought then to consider the present state of the universe as the effect of its previous state and as the cause of that which is to follow. An intelligence that, at a given instant, could comprehend all the forces by which nature is animated and the respective situation of the beings that make it up, if moreover it were vast enough to submit these data to analysis, would encompass in the same formula the movements of the greatest bodies of the universe and those of the lightest atoms. For such an intelligence nothing would be uncertain, and the future, like the past, would be open to its eyes.

The fact that this quotation comes from a (fundamental) book on probability theory shows that Laplace's view on determinism was far from naive [38]. We lack the "vast intelligence" he mentions, so we are forced to use probabilities to understand dynamical systems. Isn't that a modern idea, the first reference to ergodic theory?

In his little book "Matter and Motion" published in 1876, Maxwell insists on the sensitivity to initial conditions in physical phenomena: the intelligence mentioned by Laplace must indeed be infinitely vast [48]! One should notice that, according to Maxwell, this sensitivity is not the common rule, but rather and exception. This debate is still not really closed today. 
There is a maxim which is often quoted, that "The same causes will always produce the same effects".

To make this maxim intelligible we must define what we mean by the same causes and the same effects, since it is manifest that no event ever happens more that once, so that the causes and effects cannot be the same in all respects.

$[\ldots]$

There is another maxim which must not be confounded with that quoted at the beginning of this article, which asserts "That like causes produce like effects".

This is only true when small variations in the initial circumstances produce only small variations in the final state of the system. In a great many physical phenomena this condition is satisfied; but there are other cases in which a small initial variation may produce a great change in the final state of the system, as when the displacement of the "points" causes a railway train to run into another instead of keeping its proper course.

With his sense of eloquence, Poincaré expresses in 1908 the dependence to initial conditions in a way almost as fashionable as Lorenz's butterfly that we will describe below, including the devastating cyclone [62]:

Why have meteorologists such difficulty in predicting the weather with any certainty? Why is it that showers and even storms seem to come by chance, so that many people think it quite natural to pray for rain or fine weather, though they would consider it ridiculous to ask for an eclipse by prayer? We see that great disturbances are generally produced in regions where the atmosphere is in unstable equilibrium. The meteorologists see very well that the equilibrium is unstable, that a cyclone will be formed somewhere, but exactly where they are not in a position to say; a tenth of a degree more or less at any given point, and the cyclone will burst here and not there, and extend its ravages over districts it would otherwise have spared. If they had been aware of this tenth of a degree they could have known it beforehand, but the observations were neither sufficiently comprehensive nor sufficiently precise, and that is the reason why it all seems due to the intervention of chance.

Poincaré's second quotation shows that he does not consider chaos as an obstacle to a global understanding of the dynamics [61]. However, the context shows that he is discussing gas kinetics, which depends on a huge number of degrees of freedom (positions and speeds of all atoms). Even though Poincaré has realized the possibility of chaos in celestial mechanics (which depends on much fewer degrees of freedom), he has apparently not proposed to use probabilistic methods to study it ${ }^{4}$.

\footnotetext{
${ }^{4}$ Except for the recurrence theorem?
} 
You are asking me to predict future phenomena. If, quite unluckily, I happened to know the laws of these phenomena, I could achieve this goal only at the price of inextricable computations, and should renounce to answer you; but since I am lucky enough to ignore these laws, I will answer you straight away. And the most astonishing is that my answer will be correct.

Could this type of ideas apply to celestial mechanics as well? In his 1898 article on the geodesics of surfaces of negative curvature, after noticing that "a tiny change of direction of a geodesic [...] is sufficient to cause any variation of the final shape of the curve", Hadamard concludes in a cautious way [34].

Will the circumstances we have just described occur in other problems of mechanics? In particular, will they appear in the motion of celestial bodies? We are unable to make such an assertion. However, it is likely that the results obtained for these difficult cases will be analogous to the preceding ones, at least in their degree of complexity.

$[\ldots]$

Certainly, if a system moves under the action of given forces and its initial conditions have given values in the mathematical sense, its future motion and behavior are exactly known. But, in astronomical problems, the situation is quite different: the constants defining the motion are only physically known, that is with some errors; their sizes get reduced along the progresses of our observing devices, but these errors can never completely vanish.

Some people have interpreted these difficulties as a sign of disconnection between mathematics and physics. On the opposite, as Duhem already noticed in 1907 , they can be seen as a new challenge for mathematics, namely the challenge to develop what he called the "mathematics of approximation" [23].

One cannot go through the numerous and difficult deductions of celestial mechanics and mathematical physics without suspecting that many of these deductions are condemned to eternal sterility.

Indeed, a mathematical deduction is of no use to the physicist so long as it is limited to asserting that a given rigorously true proposition has for its consequence the rigorous accuracy of some such other proposition. To be useful to the physicist, it must still be proved that the second proposition remains approximately exact when the first is only approximately true. And even that does not suffice. The range of these two approximations must be delimited; it is necessary to fix the limits of error which can be made in the result when the degree of precision of the methods of measuring the data is known; it is necessary to define the probable error that can be granted the data when we wish to know the result within a definite degree of approximation.

Such are the rigorous conditions that we are bound to impose on mathematical deduction if we wish this absolutely precise language to be 
able to translate without betraying the physicist's idiom, for the terms of this latter idiom are and always will be vague and inexact like the perceptions which they are to express. On these conditions, but only on these conditions, shall we have a mathematical representation of the approximate.

But let us not be deceived about it; this "mathematics of approximation" is not a simpler and cruder form of mathematics. On the contrary, it is a more thorough and more refined form of mathematics, requiring the solution of problems at times enormously difficult, sometimes even transcending the methods at the disposal of algebra today.

Later I will describe Lorenz's fundamental article, dating back to 1963, which bears the technical title "Deterministic non periodic flow", and was largely unnoticed by mathematicians during about 10 years [44]. In 1972 Lorenz gave a conference entitled "Predictability: does the flap of a butterfly's wings in Brazil set off a tornado in Texas?", which made famous the butterfly effect [45]. The three following sentences, extracted from this conference, seem to me quite remarkable.

If a single flap of a butterfly's wing can be instrumental in generating a tornado, so all the previous and subsequent flaps of its wings, as can the flaps of the wings of the millions of other butterflies, not to mention the activities of innumerable more powerful creatures, including our own species.

If a flap of a butterfly's wing can be instrumental in generating a tornado, it can equally well be instrumental in preventing a tornado.

More generally, I am proposing that over the years minuscule disturbances neither increase nor decrease the frequency of occurrence of various weather events such as tornados; the most they may do is to modify the sequence in which these events occur.

The third sentence in particular is scientifically quite deep, since it proposes that the statistical description of a dynamical system could be unsensitive to the initial conditions; this idea could be seen as a precursor of the Sinai-Ruelle-Bowen measures which, as I will later describe, provide a quantitative description of this type of chaotic system.

\section{The old paradigm of periodic orbits}

\subsection{Some jargon}

One should first set up the jargon of the theory of dynamical systems. The spaces on which the motion takes place will almost always be the numerical spaces $\mathbb{R}^{n}$ (and most often $\mathbb{R}^{3}$ ). Sometimes more general spaces, like differentiable manifolds $V$ (e.g., a sphere or a torus) will represent the phase space of the system. The topology of $V$ and the dynamics can be strongly correlated, this interaction being the main motivation for Poincaré to study topology. However, we will ignore this aspect here ... 
The dynamics is generated by an evolution differential equation, or equivalently a vector field $X$ (assumed differentiable) on $V$. Each point $x$ on $V$ is the starting point of a trajectory (or an orbit) on $X$. Without any further assumption, this trajectory may not be defined for all time $t \in \mathbb{R}$ : it could escape to infinity in finite time. If all trajectories are defined for for all $t \in \mathbb{R}$, the field is said to be complete: this is always the case if $V$ is compact. In this case, for $x \in V$ and $t \in \mathbb{R}$, we may denote by $\phi^{t}(x)$ the position at time $t$ of the trajectory starting at $x$ for $t=0$. For each $t \in \mathbb{R}$ the transformation $\phi^{t}: x \mapsto \phi^{t}(x)$ is a differentiable bijection on $V$ (with differentiable inverse $\phi^{-t}$ ): it is a diffeomorphism of $V$. Obviously $\phi^{t_{1}+t_{2}}$ is the composition of $\phi^{t_{1}}$ and $\phi^{t_{2}}$, and $\left(\phi^{t}\right)_{t \in \mathbb{R}}$ is called the flow generated by the vector field $X$. In other terms, $\phi^{t}$ represents the "vast intelligence" Laplace was dreaming of, which would potentially solve all differential equations! When a mathematician writes "Let $\phi^{t}$ be the flow generated by $X$ ", he claims he has made this dream real... Of course, in most cases we make as if we knew $\phi^{t}$, but in reality knowing only asymptotic behavior when $t$ goes to infinity would be sufficient for us.

One could object - with some ground - that the evolution of a physical system has no reason to be autonomous, namely the vector field $X$ could itself depend on time. This objection is, in some way, at the heart of Maxwell's argument, when he notices that the same cause can never occur at two different times, because indeed the times are different. I will nevertheless restrict myself to autonomous differential equations, because they cover a sufficiently vast range of applications, and also because it would be impossible to develop such a rich theory without any assumption on the time dependence of the vector field.

The vector fields on $\mathbb{R}^{n}$ are not always complete, but they may be transverse to a sphere, such that the orbits of points on the sphere enter the ball and can never exit. The trajectories of points in the ball are then well defined for all $t \geqslant 0$. In this case, $\phi^{t}$ is in fact only a semiflow of the ball, in the sense that it is defined only for $t \geqslant 0$; this is not a problem if one is only interested in the future of the system. The reader not familiar with differential topology can, as a first step, restrict himself to this particular case.

It is customary to study as well discrete time dynamics. One then chooses a diffeomorphism $\phi$ on a manifold and studies its successive iterations $\phi^{k}=\phi \circ \phi \circ$ $\cdots \circ \phi$ ( $k$ times) where the time $k$ is now an integer.

One can often switch between these two points of view. From a diffeomorphism $\phi$ on $V$, one can glue together the two boundaries of $V \times[0,1]$ using $\phi$, such as to construct a manifold $\tilde{V}$ with one dimension more than $V$, which carries a natural vector field $X$, namely $\frac{\partial}{\partial s}$, where $s$ is the coordinate on $[0,1]$ (see Figure 2). Notice that the vector field $X$ is nonsingular. The original manifold $V$ can be considered as a hypersurface in $\tilde{V}$, transverse to $X$, for instance given by fixing $s=0 . V$ is often called a global section of $X$ in $\tilde{V}$. The orbits $\phi^{t}(x)$ of $X$ travel inside $\tilde{V}$ and meet the global section $V$ when $t$ is integral, on the orbits of the diffeomorphism $\phi$. Clearly, the continuous time flow $\phi^{t}$ on $\tilde{V}$ and the discrete 

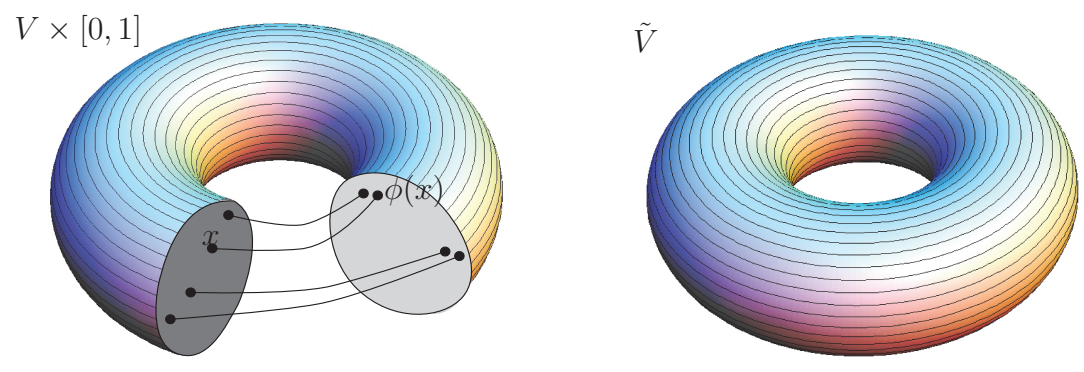

Figure 2. A suspension

time dynamics $\phi^{k}$ on $V$ are so closely related to each other, that understanding the latter is equivalent with understanding the former. The flow $\phi^{t}$, or the field $X$, is called the suspension of the diffeomorphism $\phi$.

Conversely, starting from a vector field $X$ on a manifold $\tilde{V}$, it is often possible to find a hypersurface $V \subset \tilde{V}$ which meets each orbit infinitely often. To each point $x \in V$ one can then associate the point $\phi(x)$, which is the first return point on $V$ along the future orbit of $x$. This diffeomorphism $\phi$ on $V$ is the first return map. The field $X$ is then the suspension of the diffeomorphism $\phi .^{5}$

Yet all fields are not suspensions. In particular, vector fields with singularities cannot be suspensions, and we will see that this is the case of the Lorenz equation... Nevertheless, this idea (due to Poincaré) to transform a continuous dynamics into a discrete one is extremely useful, and can be adapted, as we will see later.

\subsection{A belief}

For a long time, one has focussed on two types of orbits:

- the singularities of $X$ are fixed points of the flow $\phi^{t}$ : these are equilibrium positions of the system;

- the periodic orbits are the orbits of points $x$ such that, for some $T>0$ one has $\phi^{T}(x)=x$.

The old paradigm which entitles this section is the belief that, in general, after a transient period, the motion evolves into a permanent régime which is either an equilibrium, or a periodic orbit.

Let us be more precise. For $x$ a point in $V$, one defines the $\omega$-limit set (resp. the $\alpha$-limit set) of $x$, as the set $\omega_{X}(x)$ (resp. $\alpha_{X}(x)$ ) made of the accumulation points of the orbit $\phi^{t}(x)$ when $t \rightarrow+\infty$ (resp. $\left.t \rightarrow-\infty\right)$. The above belief consists in the statement that, for a "generic" vector field $X$ on $V$, and for each $x \in V$, the set $\omega_{X}(x)$ reduces either to a single point, or to a periodic orbit. It is not necessary to insist on the ubiquity of periodic phenomena in science (for instance in

${ }^{5}$ In general the return time is not necessarily constant, and the flow needs to be reparametrized to obtain a true suspension. 
astronomy). The realization that other types of régimes could be expected occurred amazingly late in the history of science.

In fact, the first fundamental results in the theory of dynamical systems, around the end of the nineteenth century, had apparently confirmed this belief. Let us cite for instance the Poincaré-Bendixson theorem: for any vector field on the two-dimensional disk, entering the disk on the boundary and possessing (to simplify) finitely many fixed points, the $\omega$-limit sets can only be of three types: a fixed point, a periodic orbit or a singular cycle (that is, a finite number of singular points connected by finitely many regular orbits). See Figure 3.
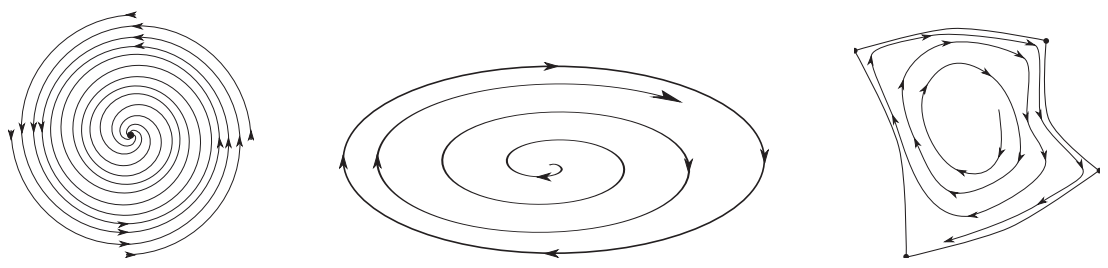

Figure 3. A fixed point, a limiting periodic orbit, and a "cycle".

It is easy to show that the case of a singular cycle is "unstable", that is, it does not occur for a "generic" vector field; this remark confirms the above belief, in the case of flows on the disk. Interestingly, Poincaré did not explicitly notice the (rather simple) fact that, among the three asymptotic limits, one is "exceptional", while the two others are "generic". Robadey's thesis [63] discusses the concepts of genericity in Poincaré's work. The explicit investigation of the behavior of generic vector fields started much later, probably with Smale and Thom at the end of the 1950 s.

The case of vector fields on general surfaces needed more work, and was only completed in 1962 by Peixoto [55]. There is no Poincaré-Bendixson type theorem, and the $\omega$-limit sets can be much more complicated than for the disk. Yet, these complex examples happen to be "rare", and generally the limit sets are indeed equilibrium points or periodic orbits.

We shall now introduce an important concept, which we will later widely generalize. A singularity $x_{0}$ of a vector field $X$ (on a manifold $V$ ) is said to be hyperbolic if the linearized vector field at this point (namely, a matrix) has no eigenvalue on the imaginary axis. The set of points $x$ the orbits of which converge to $x_{0}$ is the stable manifold of $x_{0}$ : it is a submanifold $W^{s}\left(x_{0}\right)$ immersed in $V$, which contains $x_{0}$ and has dimension equal to the number of eigenvalues with negative real parts. Switching to $-X$, one similarly defines the unstable manifold $W^{u}\left(x_{0}\right)$ of $x_{0}$, of dimension complementary to that of $W^{s}\left(x_{0}\right)$ (since the singularity is hyperbolic).

If $\gamma$ is a periodic orbit containing a point $x_{0}$, one can choose a ball $B$ of dimension $n-1$ containing $x_{0}$ and transverse to the flow. The flow then allows to define Poincaré's first return map, which is a local diffeomorphism in $B$ defined 
near $x_{0}$ and fixing that point. If the linearization of this diffeomorphism at $x_{0}$ does not have any eigenvalue of modulus 1 , then the orbit $\gamma$ is said to be hyperbolic. The set of points $x$ such that $\omega_{X}(x)$ coincides with the periodic orbit $\gamma$ is the stable manifold of $\gamma$, denoted by $W^{s}(\gamma)$. As above, it is an immersed submanifold. The unstable manifold $W^{u}(\gamma)$ is defined similarly.

In 1959 Smale defined the vector fields which are now called of Morse-Smale type [72]. These fields are characterized by the following properties:

- $X$ has at most finitely many singularities and periodic orbits, which are all hyperbolic;

- The $\alpha$ and $\omega$-limit sets of all points are singularities or periodic orbits;

- the stable and unstable manifolds of the singularities or the periodic orbits intersect each other transversally.
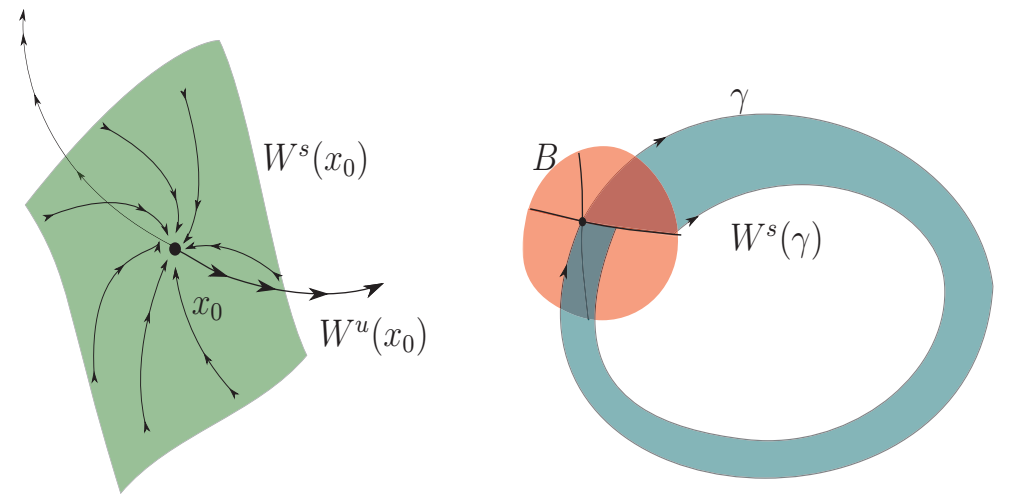

FIGURE 4. Hyperbolic fixed point and periodic orbit

In this article Smale formulated a triple conjecture. Before stating it, I first need to explain the fundamental concept of structural stability, introduced in 1937 by Andronov and Pontrjagin [5]. A vector field $X$ is structurally stable if there exists a neighborhood of $X$ (in the $C^{1}$ topology on vector fields) such that all fields $X^{\prime}$ in this neighborhood are topologically conjugate to $X$. This conjugacy means that there exists a (generally non-differentiable) homeomorphism in $V$ which maps the orbits of $X$ to the orbits of $X^{\prime}$, keeping the time orientations. For such fields $X$, the topological dynamics is qualitatively insensitive to small perturbations. This concept of structural stability thus belongs to the "mathematics of approximation" called for by Duhem. Andronov and Pontrjagin had shown that certain very simple fields on the two-dimensional disk are structurally stable. The most naive example is given by the radial field $X=-x \partial / \partial x-y \partial / \partial y$, for which all points converge towards the (singular) origin. If one perturbs $X$, the new field will still have an attracting singularity near the origin: $X$ is thus structurally stable. 
I can now state Smale's conjectures:

1. Given a compact manifold $V$, the Morse-Smale fields form an open dense set in the space of all vector fields on $V$.

2. All Morse-Smale fields are structurally stable.

3. All structurally stable fields are of Morse-Smale type.

The first and third conjecture are false, as Smale will soon himself discover. But the second one is true... Smale's motivation for these conjectures was clear: he wanted to prove the (then dominant) belief in a permanent régime - equilibrium point or periodic orbit - for a generic system.

In 1962 Peixoto proved the 3 conjectures if $V$ is a compact orientable surface [55].

The first of Smale's conjectures is surprising, since Poincaré or Birkhoff already knew it was false: a flow can have infinitely many periodic orbits in a stable way. About this period, Smale wrote in 1998 [77]:

It is astounding how important scientific ideas can get lost, even when they are aired by leading scientific mathematicians of the preceding decades.

It was explicitly realized around 1960 that the dynamics of a generic vector field is likely to be much more complicated than that of a Morse-Smale field. The paradigm of the periodic orbits yielded to the next one - that of hyperbolic systems - which I will describe in the next section. But periodic orbits will continue to play a fundamental rôle in dynamics, as Poincaré had explained in 1892 [59, Chap. 3, Sec. 36]:

In addition, these periodic solutions are so valuable for us because they are, so to say, the only breach by which we may attempt to enter an area heretofore deemed inaccessible.

One more remark before going on towards the Lorenz attractor ... The dynamics we are considering here are not assumed to be conservative. One could discuss for instance the case of Hamiltonian dynamical systems, which enjoy very different qualitative behaviors. For example, the preservation of phase space volume implies that almost all points are recurrent, as follows from Poincaré's recurrence theorem. A conservative field is thus never of Morse-Smale type.

In the domain of Hamiltonian vector fields, the old paradigm is quasi-periodic motion. One believes that in the ambient manifold, "many orbits" are situated on invariant tori supporting a linear dynamics, generated by a certain number of uncoupled harmonic oscillators with different periods. The typical example is the Keplerian motion of the planets, assuming they do not interact with each other: each planet follows a periodic trajectory, and the whole system moves on a torus of dimension given by the number of planets. The KAM theory allows to show that many of these invariant tori persist if one perturbs a completely integrable Hamiltonian system. On the other hand, there is no reason to find such tori for a "generic" Hamiltonian system. 
One should thus keep in mind that we are discussing here a priori dissipative dynamics. It is amazing that eminent physicists like Landau and Lifschitz have for a while presented turbulence as an almost periodic phenomenon, with invariant tori of dimensions depending on Reynold's number. Only in the 1971 second edition of their famous treatise on fluid mechanics have they realized that almost periodic functions are "too nice" to describe turbulence.

\section{Second attempt: hyperbolic dynamics}

\subsection{Hadamard and the geodesics on a bull's forehead}

In 1898 Hadamard publishes a remarkable article on the dynamical behavior of the geodesics on surfaces of negative curvature [34]. This article can be considered as the starting point of the theory of hyperbolic dynamical systems and of symbolic dynamics. It probably appeared too early since, more than 60 years later, Smale had to follow again the same path followed by Hadamard before continuing much further, as we will see.
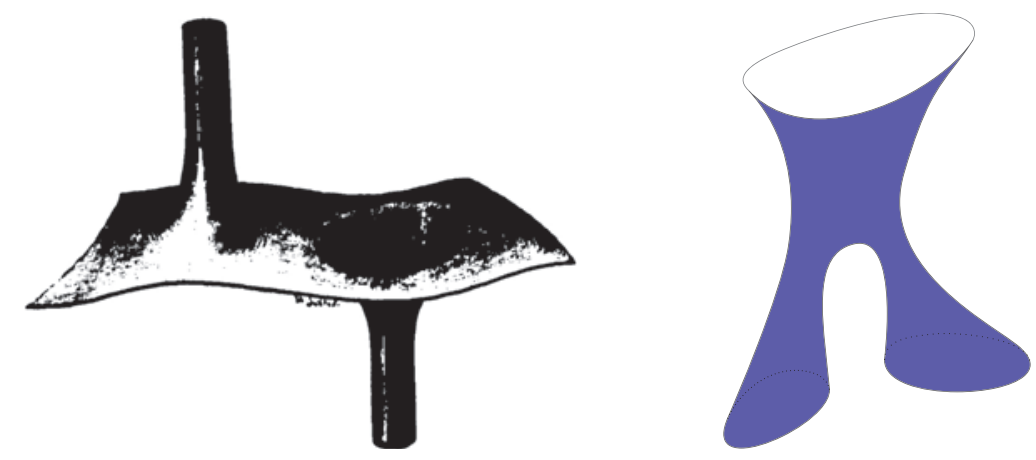

FiguRE 5. Hadamard's pants

Hadamard starts by giving some concrete examples of negatively curved surfaces in the ambient space. The left part of Figure 5 is extracted from his article. This surface is diffeomorphic to a plane minus two disks, which allows me to call it "(a pair of) pants", and to draw it like on the right part of Figure 5.

The problem is to understand the dynamical behavior of the geodesics on this surface. That is, a point is constrained to move on $P$, only constrained by the reaction force. At each moment the acceleration is orthogonal to the surface: the trajectories are geodesics of $P$, at constant speed.

An initial condition consists in a point on $P$ and a tangent vector to $P$ on this point, say of length 1 . The set of these initial conditions forms the manifold $V=T^{1} P$ of dimension 3, called the unitary tangent bundle of $P$. The geodesic flow $\phi^{t}$ acts on $V$ : one considers the geodesic starting from a point in a certain direction, and follow it during the time $t$ to get another point and another direction. 
The main property of the negative curvature used by Hadamard is the following: every continuous path drawn on the surface between two points can be deformed (keeping the boundary points fixed) into a unique geodesic arc joining the two boundary points.

One can find three closed geodesics $g_{1}, g_{2}, g_{3}$ cutting $P$ into 4 parts. Three of them correspond to the three "ends" of $P$, and the fourth one is the convex core, which is a compact surface with boundary given by the three geodesics (see Figure 6).

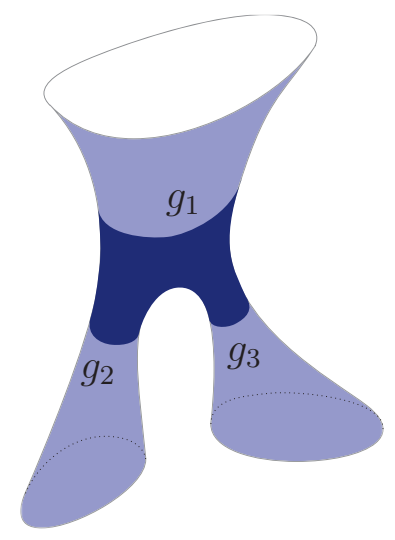

Figure 6. The core of the pants

Let us now consider a geodesic $t \in \mathbb{R} \mapsto g(t) \in P$. If the curve $g$ intersects $g_{1}, g_{2}$ or $g_{3}$ at time $t_{0}$, to exit the core and enter one end, the property I have just mentioned shows that for any $t>t_{0}$ the curve $g(t)$ remains in this end and cannot come back in the core. In fact, one can check that $g(t)$ goes to infinity in that end. Conversely, if a geodesic enters the core at time $t_{0}$, it remains in one of the ends when $t \rightarrow-\infty$. One can also show that no geodesic can stay away from the core for ever. There are thus several types of geodesics:

- $g(t)$ is in the core for all $t \in \mathbb{R}$;

- $g(t)$ comes from one end, enters the core and exits into an end;

- $g(t)$ comes from one end, enters the core and stays there for all large $t$;

- $g(t)$ is in the core for $t$ sufficiently negative, and exits into an end.

The most interesting ones are of the first type: they are called nowadays nonwandering orbits. Hadamard analyzed them as follows. Let us join $g_{1}$ and $g_{2}$ by an arc $c_{3}$ of minimal length: it is a geodesic arc inside the core, orthogonal to $g_{1}$ and $g_{2}$. Similarly, let $c_{1}$ connect $g_{2}$ with $g_{3}$, and $c_{2}$ connect $g_{1}$ with $g_{3}$. The arcs $c_{i}$ are the seams of the pants. If one cuts the core along the seams, one obtains two hexagons $H_{1}$ and $H_{2}$ (see Figure 7).

Let us now consider a geodesic $g$ of the first type, that is entirely contained in the core. The point $g(0)$ belongs to one of the hexagons, maybe to both if it lies 

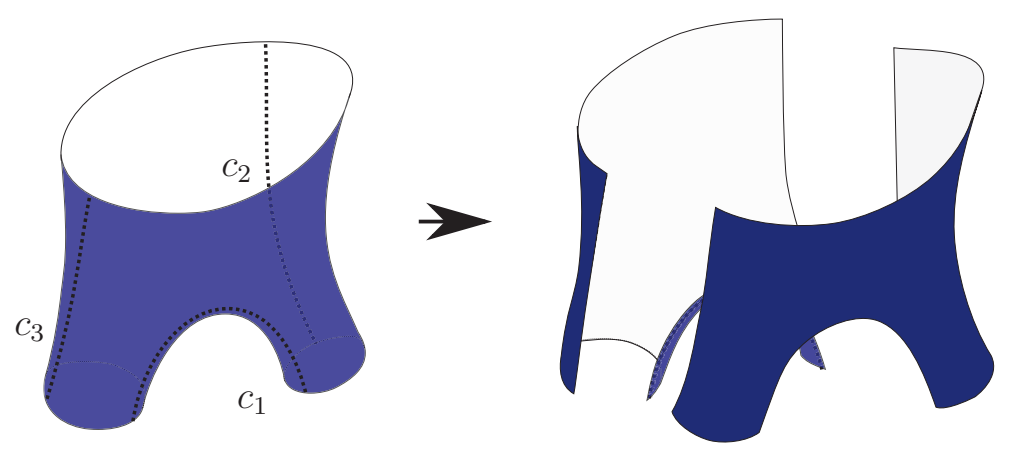

FiguRE 7. Three cuts in the pants leading to two hexagons

on a seam, but let us ignore this particular case. When one follows the geodesic $g$ from $g(0)$ by increasing the time $t$, one successively intersects the seams $c_{1}, c_{2}, c_{3}$ infinitely many times ${ }^{6}$. One can then read an infinite word written in the 3 -letter alphabet $\{1,2,3\}$. If $g$ intersects the seam $c_{1}$, the next seam cannot be $c_{1}$ : it will be either $c_{2}$ or $c_{3}$. The word associated to a geodesic will thus never contain two successive identical letters.

Similarly, one can follow $g$ in the negative time direction and obtain a word. These two words make up a single bi-infinite word $m(g)$ associated with the geodesic $g$.

Actually, Hadamard only treats the closed geodesics, which are associated with the periodic words. In 1923 Morse will complete the theory by coding the nonperiodic geodesics by bi-infinite words [50]. In the sequel I will mix the two articles, and call their union Hadamard-Morse.

The main result of Hadamard-Morse is the following:

For any bi-infinite word $m$ in the alphabet $\{1,2,3\}$ without repetition, there exists a geodesic $g$ realizing that word. The geodesic $g$ is unique if one specifies the hexagon containing $g(0)$.

Of course, the uniqueness of the geodesic should be understood as follows: one can move the origin of $g$ into $g(\tau)$ without changing the word, as long as $g$ does not meet any seam between $t=0$ and $t=\tau$. Uniqueness means that if two geodesics of type 1 are associated with the same word and start from the same hexagon, then they can only differ by such a (short) time shift.

The proof of this result is quite easy (with modern techniques). Starting from a bi-infinite word, one takes the word $m_{p}$ of length $2 p$ obtained by keeping only the $p$ first letters on the right and on the left. One then considers a path $\gamma_{p}:\left[-l_{p}, l_{p}\right] \mapsto P$ which "follows" the word $m_{p}$ and starts from $H_{1}$ or $H_{2}$. More precisely, one chooses a point $x_{1}$ in $H_{1}$ and $x_{2}$ in $H_{2}$, and the path $\gamma_{p}$ is formed by $2 p$ geodesic arcs alternatively joining $x_{1}$ and $x_{2}$ and crossing the seams as indicated

${ }^{6}$ This fact is due to the negative curvature. 
in the word $m_{p}$. This arc $\gamma_{p}$ can be deformed, keeping the boundaries fixed, into a geodesic arc $\tilde{\gamma}_{p}:\left[-\bar{l}_{p}, \bar{l}_{p}\right] \rightarrow P$. One then needs to show that this path converges to a geodesic $g: \mathbb{R} \rightarrow P$ when $p \rightarrow \infty$, which is easy for a modern mathematician (using Ascoli's theorem etc.). Uniqueness is not very difficult to check either.

Here are a few qualitative consequences:

- If two bi-infinite words $m$ and $m^{\prime}$ coincide from a certain index on, the corresponding geodesics $g, g^{\prime}$ will approach each other when $t \rightarrow+\infty$ : they are asymptotes. There exists $\tau$ such that the distance between $g(t)$ and $g^{\prime}(t+\tau)$ tends to 0 when $t \rightarrow+\infty$. Nowadays we would say that $g$ and $g^{\prime}$ belong to the same stable manifold. Of course, a similar remark applies when $m$ and $m^{\prime}$ coincide for all sufficiently negative indices: the geodesics then belong to the same unstable manifold.

- Starting from two infinite words $m_{+}$and $m_{-}$without repetitions, one can of course construct a bi-infinite word $m$ coinciding with $m_{+}$for sufficiently positive indices, and with $m_{-}$for sufficiently negative indices. Hence, given two nonwandering geodesics, one can always find a third one which is an asymptote of the first one in the future, and an asymptote of the second one in the past. One can even arbitrarily fix any finite number of indices of $m$. Obviously, this result implies a sensitive dependence to the initial conditions: an arbitrarily small perturbation of a given geodesic can make it asymptote to two arbitrary geodesics, respectively in the past and in the future.

- Another interesting property: any nonwandering geodesic can be approached arbitrarily close by periodic geodesics. Starting from a bi-infinite word, it suffices to consider a long segment of this word and to repeat it infinitely often to construct a nearby periodic geodesic. Therefore there exist countably many periodic geodesics, and their union is dense in the set of nonwandering points. The dynamical behavior of these geodesics is thus much more complicated than for a Morse-Smale flow.

To end this brief account of these two articles, I shall mention some consequences Hadamard-Smale could have "easily" obtained, but did not notice.

- The description of the dynamics of the geodesics does not depend on the choice of metric with negative curvature. One could "almost" deduce that for two metrics of negative curvature on $P$, the geodesic flows are topologically conjugate. We are close to the structural stability, proved by Anosov in 1962 [3]: the geodesic flow of a Riemannian metric with negative curvature is structurally stable. Moreover, Gromov showed that, on a given compact manifold, the geodesic flows of two arbitrary metrics of negative curvature are topologically conjugate [30]. ${ }^{7}$

- Another aspect, implicit in these articles, is the introduction of a dynamics on a space of symbols. The space of all sequences $\left(x_{i}\right)_{i \in \mathbb{Z}} \in\{1,2,3\}^{\mathbb{Z}}$ is compact, and the shift of the indices $\left(x_{i}\right) \mapsto\left(x_{i+1}\right)$ is a homeomorphism which "codes" the dynamics of the geodesic flow. More precisely, one should take the "subshift of

\footnotetext{
${ }^{7}$ Actually, the two metrics could be defined on two different manifolds with isomorphic fundamental groups.
} 


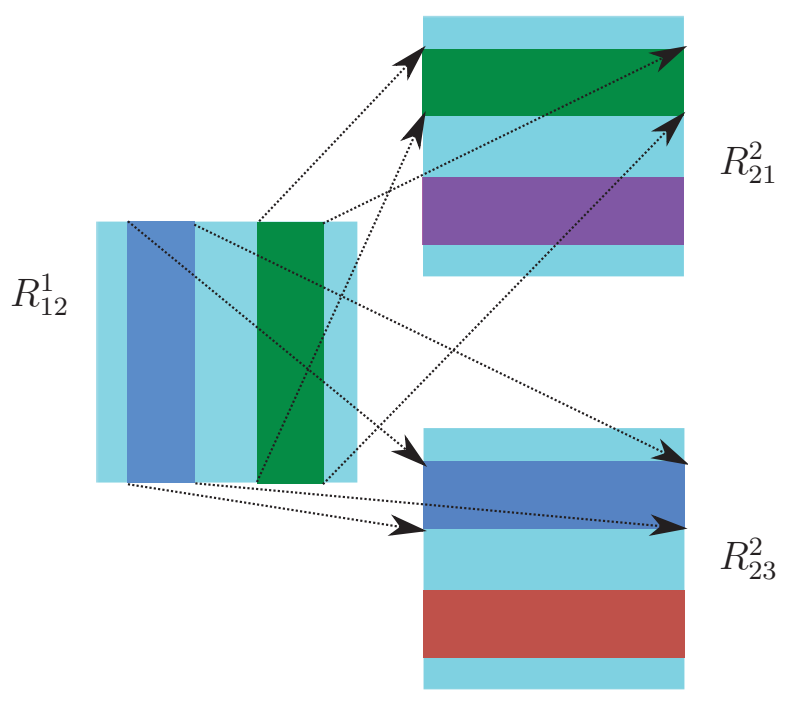

FiguRE 8. Dynamics on the rectangles $R_{i j}$

finite type" formed by the sequences without repetitions, and (even more precisely) one should take two copies of this space, corresponding to the two hexagons.

- Finally, by extrapolating a little, one could see in these articles a subliminal construction of Smale's horseshoe, an object I will soon describe. Indeed, consider all unit vectors tangent to $P$ on a point of a seam, heading towards another seam. The geodesic generated by such an initial condition starts from $c_{i}$, crosses $H_{1}$ or $H_{2}$, and lands on $c_{j}$. These unit vectors form twelve rectangles: one has to choose $c_{i}, c_{j}$, then $H_{1}$ or $H_{2}$, which gives twelve possibilities, and for each one we should indicate the starting and arrival points on the seams. These twelve rectangles denoted by $R_{i j}^{1}, R_{i j}^{2}$ - are embedded in $T^{1} P$, the unitary tangent bundle of $P$, transversely to the geodesic flow.

Strictly speaking, the union $R$ of these rectangles is not a global section for the geodesic flow, since they do not meet all the geodesics: the seams themselves define geodesics not meeting $R$. Nevertheless, one can define a "first return" map $\phi$, which is not defined in the whole of $R$, and which is not surjective. Starting from a unit vector $x$ in $R$, the corresponding geodesic crosses a hexagon and lands on another cut. The tangent vector at the exit point is not necessarily in $R$, since the geodesic could then exit the second hexagon along $g_{1}, g_{2}$ or $g_{3}$ instead of another cut. If this tangent vector is still in $R$, we denote it by $\phi(x)$. The domain of definition of $\phi$ is the union of 24 "vertical rectangular" zones, two in each rectangle, and its image is formed by 24 "horizontal rectangular" zones. Each of the 24 vertical rectangular zones is contracted by $\phi$ along the vertical direction, and expanded along the horizontal direction, and its image is one of the 24 horizontal 
rectangular zones. Figure 8 displays the two vertical zones in $R_{12}^{1}$ and their images in $R_{21}^{2}$ and $R_{23}^{2}$.

This simultaneous appearance of expansion and contraction is clearly at the heart of the phenomenon, but it was not explicitly noticed by Hadamard-Morse. An easy observation: if one slightly perturbs the geodesic flow (not necessarily into another geodesic flow), $R$ will still be transverse to the flow, and the "return" on $R$ will have the same shape, with slightly deformed rectangular zones; the nonwandering orbits will still be coded by sequences of symbols, accounting for the sequences of rectangles crossed along the evolution. The structural stability (at least of the nonwandering set) can be easily deduced from this observation.

It is surprising that this article of Hadamard, containing so many original ideas, could stay unnoticed so long. Yet, Duhem described this article in such a "colorful" way as to attract the attention even of non-mathematicians [23].

Imagine the forehead of a bull, with the protuberances from which the horns and ears start, and with the collars hollowed out between these protuberances; but elongate these horns and ears without limit so that they extend to infinity; then you will have one of the surfaces we wish to study. On such a surface geodesics may show many different aspects. There are, first of all, geodesics which close on themselves. There are some also which are never infinitely distant from their starting point even though they never exactly pass through it again; some turn continually around the right horn, others around the left horn, or right ear, or left ear; others, more complicated, alternate, in accordance with certain rules, the turns they describe around one horn with the turns they describe around the other horn, or around one of the ears. Finally, on the forehead of our bull with his unlimited horns and ears there will be geodesics going to infinity, some mounting the right horn, others mounting the left horn, and still others following the right or left ear. [...] If, therefore, a material point is thrown on the surface studied starting from a geometrically given position with a geometrically given velocity, mathematical deduction can determine the trajectory of this point and tell whether this path goes to infinity or not. But, for the physicist, this deduction is forever useless. When, indeed, the data are no longer known geometrically, but are determined by physical procedures as precise as we may suppose, the question put remains and will always remain unanswered.

\subsection{Smale and his horseshoe}

Smale has given several accounts of his discovery of hyperbolic systems around $1960^{8}$ (see for instance [77]). This discovery is independent of the previous contributions of Poincaré and Birkhoff, which however played a rôle in the subsequent developments of the theory. It seems that Hadamard's article played absolutely

\footnotetext{
${ }^{8} \mathrm{On}$ the beach in Copacabana, more precisely in Leme!
} 
no rôle at this time. Nevertheless, Smale constructs a counterexample to his own conjecture, according to which Morse-Smale flows form an open dense set in the space of dynamical systems: the famous horseshoe. It is a diffeomorphism $\phi$ on the two-dimensional sphere $\mathbb{S}^{2}$, thought of as the plane $\mathbb{R}^{2}$ with an extra point at infinity. $\phi$ is assumed to map a rectangle $R$ in the plane as shown on Figure 9 , by expanding the vertical directions and contracting the horizontal ones. The intersection $R \cap \phi(R)$ is the union of two rectangles $R_{1}, R_{2}$.

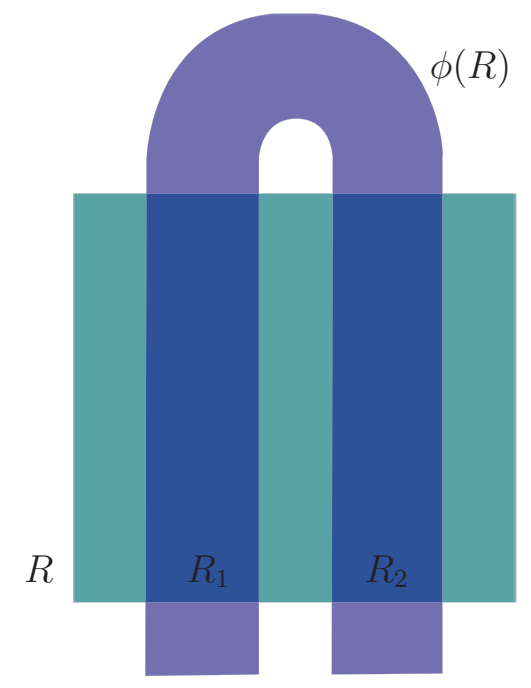

Figure 9. Smale's horseshoe

One can assume that the point at infinity is a repulsive fixed point. The points of $R$ which always stay in $R$, that is $\cap_{k \in \mathbb{Z}} \phi^{k}(R)$, form a Cantor set, homeomorphic to $\{1,2\}^{\mathbb{Z}}$. Each of its points is coded by the sequence of rectangles $R_{1}, R_{2}$ successively visited by its orbit. In particular, the periodic points form an infinite countable set, dense in this Cantor set. Smale then establishes that the horseshoe is structurally stable, a rather easy fact (nowadays). If one perturbs the diffeomorphism $\phi$ into a diffeomorphism $\phi^{\prime}$, the intersection $\phi^{\prime}(R) \cap R$ is still made of two "rectangular" zones crossing $R$ all along, and one can still associate a single orbit to each sequence in $\{1,2\} \mathbb{Z}$, allowing to construct a topological conjugacy between $\phi$ and $\phi^{\prime}$, at least on these invariant Cantor sets. There just remains to extend the conjugacy in the exterior, using the fact that all exterior orbits come from infinity.

Smale publishes this result in the proceedings of a workshop organized in the Soviet Union in 1961 [73]. Anosov tells us about this "hyperbolic revolution" in $[4]$.

The world turned upside down for me, and a new life began, having read Smale's announcement of "a structurally stable homeomorphism with an infinite number of periodic points", while standing in line to register for 
a conference in Kiev in 1961. The article is written in a lively, witty, and often jocular style and is full of captivating observations. [...] [Smale] felt like a god who is to create a universe in which certain phenomena would occur.

Afterwards the theory progresses at a fast pace. The horseshoe is quickly generalized by Smale (see for instance [74]). As I already mentioned, Anosov proves in 1962 that the geodesic flow on a manifold of negative curvature is structurally stable $^{9}$. For this aim, he conceives the concept of what is known today as an Anosov flow.

A nonsingular flow $\phi^{t}$ on a compact manifold $V$, generated by a vector field $X$, is an Anosov flow if at each point $x$ one can decompose the tangent space $T_{x} V$ into three subspaces $\mathbb{R} . X(x) \oplus E_{x}^{u} \oplus E_{x}^{s}$. The first one $\mathbb{R} . X(x)$ is the line generated by the vector field, and the others are called respectively unstable and stable subspaces. This decomposition should be invariant through the differential $d \phi^{t}$ of the flow, and there should exist constants $C>0, \lambda>0$ such that for all $t \in \mathbb{R}$ and $v_{u} \in E_{x}^{u}, v_{s} \in E_{x}^{s}$ :

$$
\left\|d \phi^{t}\left(v_{u}\right)\right\| \geqslant C^{-1} \exp (\lambda t)\left\|v_{u}\right\| \quad ; \quad\left\|d \phi^{t}\left(v_{s}\right)\right\| \leqslant C \exp (-\lambda t)\left\|v_{s}\right\|
$$

(here $\|\cdot\|$ is an auxiliary Riemannian metric).

Starting from the known examples of structurally stable systems (MorseSmale, horseshoe, geodesic flow on a negatively curved manifold and a few others), Smale cooked up in 1965 the fundamental concept of dynamical systems satisfying the Axiom A (by contraction, Axiom A systems).

Consider a compact set $\Lambda \subset V$ invariant through a diffeomorphism $\phi . \Lambda$ is said to be a hyperbolic set if the tangent space to $V$ restricted to points of $\Lambda$ admits a continuous decomposition into a direct sum: $T_{\Lambda} V=E^{u} \oplus E^{s}$, invariant through the differential $d \phi$, and such that the vectors in $E^{u}$ are expanded, while those in $E^{s}$ are contracted. Precisely, there exists $C>0, \lambda>0$ such that, for all $v_{u} \in E^{u}, v_{s} \in E^{s}, k \in \mathbb{Z}$, one has:

$$
\left\|d \phi^{k}\left(v_{u}\right)\right\| \geqslant C^{-1} \exp (k \lambda)\left\|v_{u}\right\| \quad ; \quad\left\|d \phi^{k}\left(v_{s}\right)\right\| \leqslant C \exp (-k \lambda)\left\|v_{s}\right\| .
$$

A point in $V$ is called wandering if it has an open neighborhood $U$ disjoint from all its iterates: $\phi^{k}(U) \cap U=\emptyset$ for all $k \neq 0$. The set of nonwandering points is an invariant closed set, traditionally denoted by $\Omega(\phi)^{10}$.

By definition, $\phi$ is Axiom $A$ if $\Omega(\phi)$ is hyperbolic and if the set of periodic points is dense in $\Omega(\phi)$.

Under this assumption, if $x$ is a nonwandering point, the set $W^{s}(x)$ (resp. $\left.W^{u}(x)\right)$ of the points $y$ such that the distance between $\phi^{k}(y)$ and $\phi^{k}(x)$ goes to 0 when $k$ tends to $+\infty$ (resp. $-\infty$ ) is a submanifold immersed in $V$ : it is the stable (resp. unstable) manifold of the point $x$. An Axiom A diffeomorphism satisfies the strong transversality assumption if the stable manifolds are transverse to the unstable ones.

\footnotetext{
${ }^{9}$ Surprisingly, he does not seem to know Hadamard's work.

${ }^{10}$ Although the notation looks like one of an open set.
} 
An analogous definition can be given for vector fields $X$ : one then needs to continuously decompose $T_{\Lambda} V$ into a sum $\mathbb{R} . X \oplus E^{u} \oplus E^{s}$. In particular, this definition implies that the singular points of $X$ are isolated in the nonwandering set, otherwise the dimensions of the decomposition would be discontinuous.

Smale then states three conjectures, parallel to the ones he had formulated nine years earlier:

1. Given a compact manifold $V$, the Axiom A diffeomorphisms satisfying the strong transversality condition form an open dense set in the set of diffeomorphisms of $V$.

2. The Axiom A diffeomoprhisms satisfying the strong transversality condition are structurally stable

3. The structurally stable diffeomorphisms are Axiom $A$ and satisfy the strong transversality condition.

The second conjecture is correct, as shown by Robbin in 1971 and by Robinson in 1972 (assuming diffeomorphisms of class $C^{1}$ ). One had to wait until 1988 and Mañé's proof to check the third conjecture.

However, the first conjecture is wrong, as Smale himself will show in 1966 [75]. As we will see, a counterexample was actually "available" in Lorenz's article four years earlier, but it took time to the mathematics community to notice this result. Smale's counterexample is of different nature: he shows that a defect of transversality between the stable and unstable manifolds can sometimes not be "repaired" through a small perturbation. However, Smale introduces a weaker notion than structural stability, called $\Omega$-stability: it requires that, after a perturbation of the diffeomorphism $\phi$ into $\phi^{\prime}$, the restrictions of $\phi$ to $\Omega(\phi)$ and of $\phi^{\prime}$ to $\Omega\left(\phi^{\prime}\right)$ be conjugate through a homeomorphism. He conjectures that property to be generic.

Smale's 1967 article Differential dynamical systems represents an important step for the theory of dynamical systems [76], a "masterpiece of mathematical literature" according to Ruelle [69].

But, already in 1968, Abraham and Smale found a counterexample to this new conjecture, also showing that Axiom A is not generic [1]. In 1972, Shub and Smale experiment another concept of stability [70], which will lead Meyer to the following comment:

In the never-ending quest for a solution of the yin-yang problem more and more general concepts of stability are proffered.

Bowen's 1978 review article is interesting on several points [18]. The theory of Axiom A systems has become solid and, although difficult open questions remain, one has a rather good understanding of their dynamics, both from the topological and ergodic points of view. Even if certain "dark swans" have appeared in the landscape, destroying the belief in the genericity of Axiom A, these are still studied, at that time, "as if they were hyperbolic". Here are the first sentences of Bowen's article, illustrating this point of view.

These notes attempt to survey the results about Axiom A diffeomorphisms since Smale's well-known paper of 1967. In that paper, Smale 
defined these diffeomorphisms and set up a program for dynamical systems centered around them. These examples are charming in that they display complicated behavior but are still intelligible. This means that there are many theorems and yet some open problems. [...] The last sections deal with certain non-Axiom A systems that have received a good deal of attention. These systems display a certain amount of Axiom A behavior. One hopes that further study of these examples will lead to the definition of a new and larger class of diffeomorphisms, with the Axiom A class of prototype.

It is not possible here to seriously present the theory of hyperbolic systems, the reader may try [36]. I will still describe a bit later some of the most important theorems, which give a more precise account of the ergodic behavior of these systems.

Still, I have to cite one of the major results - due to Bowen - which allows to understand the dynamics à la Hadamard. Assume one covers the nonwandering set $\Omega(\phi)$ by finitely many "boxes" $B_{i}(i=1,2, \ldots, l)$, assumed compact with disjoint interiors. Let us construct a finite graph, with vertices indexed by $i$, and such that each oriented link connects $i$ to $j$ if the interior of $\phi\left(B_{i}\right) \cap B_{j}$ is nonempty (as a subset of $\Omega(\phi)$ ). Let us denote by $\Sigma$ the closure of the set of sequences $\{1,2, \ldots, l\}^{\mathbb{Z}}$ corresponding to the infinite paths on the graph, namely the sequences of indices $\sigma(p)_{p \in \mathbb{Z}}$ such that two consecutive indices are connected in the graph. One says that $\Sigma$ is a subshift of finite type. The collection of boxes is a Markov partition if this subshift faithfully codes the dynamics of $\phi$. One requires that for each sequence $\sigma \in \Sigma$, there exists a unique point $x=\pi(\sigma)$ in $\Omega(\phi)$, the orbit of which precisely follows the itinerary $\sigma$ : for each $k$, one has $\phi^{k}(x) \in B_{\sigma(k)}$. But one also requires that the coding $\pi: \Sigma \rightarrow \Omega(\phi)$ be as injective as possible: each fiber $\pi^{-1}(x)$ is finite and, for $x$ a generic point (in Baire's sense in $\Omega(\phi)$ ), it contains a single itinerary. Bowen establishes that the nonwandering sets of hyperbolic sets can be covered by this type of Markov partition. The usefulness of this result is clear, since it transfers a dynamical question into a combinatorial one.

Nowadays, Axiom A systems seem to occupy a much smaller place as was believed at the end of the 1970s. The hyperbolic paradigm has abandoned its dominant position ... Anosov's following quotation could probably be expanded beyond the mathematical world [4].

Thus the grandiose hopes of the 1960s were not confirmed, just as the earlier naive conjectures were not confirmed.

For a more detailed description of the "hyperbolic history" one can also read the introduction of [36] or of [54]. See also "What is ... a horseshoe" by one of the actors of the subject [69].

\subsection{How about Poincaré's infinitely thin tangle?}

I barely dare to set a doubt on Poincaré's legacy in this seminar bearing his name. Many authors claim that Poincaré is at the origin of chaos theory. His rôle is 
doubtlessly very important, but maybe not as much as is often claimed. Firstly, the idea that physical phenomena can be very sensitive to initial conditions was not totally new, as we have seen with Maxwell. Second, Poincaré's contributions on these questions seem to have been largely forgotten in the subsequent years, and have only played a minor rôle in the further development of the theory. Most of Poincaré's results were rediscovered. More essentially, the central idea that I wish to present here is that chaos theory cannot be restricted to the "powerless" statement that the dynamics is complicated: the theory must encompass methods allowing to explain the internal mechanisms of this dynamics. The following famous quotation of Poincaré illustrates this powerlessness facing the complexity of the dynamics [59, Chap. 33, Sec. 397]:

When we try to represent the figure formed by these two curves and their infinitely many intersections, each corresponding to a doubly asymptotic solution, these intersections form a type of trellis, tissue, or grid with infinitely fine mesh. Neither of the two curves must ever cut across itself again, but it must bend back upon itself in a very complex manner in order to cut across all of the meshes in the grid an infinite number of times.

The complexity of this figure is striking, and I shall not even try to draw it. Nothing is more suitable for providing us with an idea of the complex nature of the three-body problem, and of all the problems of dynamics in general, where there is no uniform integral and where the Bohlin series are divergent.

Poincaré's major contribution ${ }^{11}$ was to realize the crucial importance of homoclinic orbits. Let's consider a diffeomorphism $\phi$ on the plane, with a hyperbolic fixed point at the origin, with stable and unstable subspaces of dimension 1 . A point $x$ (different from the origin) is homoclinic if it belongs to the intersection $W^{s} \cap W^{u}$ of the stable and unstable manifolds of the origin. The orbit of $x$ converges to the origin both in the future and in the past.

It is not necessary to recall here in detail how this concept appeared in 1890. On this topic, I recommend the reading of [12], which relates the beautiful story of Poincaré's mistake in the first version of his manuscript when applying for King Oscar's prize [12]. The book [11] also discusses this prize, insisting on the historical and sociological aspects rather than the mathematical ones.

Poincaré's setup was inspired by celestial mechanics, so $\phi$ preserves the area, and he had first thought it was impossible for $W^{s}$ and $W^{u}$ to cross each other without being identical. He deduced from there a stability theorem in the three body problem. When he realized his error, he understood that these homoclinic points where $W^{s}$ meets $W^{u}$ transversely are not only possible, but actually the rule. He also understood that the existence of such a point of intersection implies that the geometry of the curves $W^{s}$ and $W^{u}$ must indeed constitute an "infinitely thin tangle". Poincaré did not try to draw the tangle, but to illustrate the complexity of the situation he shows that the existence of a transverse homoclinic

\footnotetext{
$\overline{11_{\text {in }} \text { this domain! }}$
} 


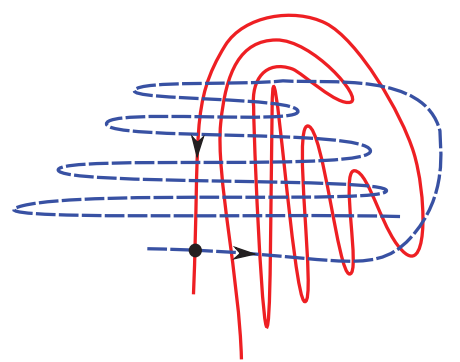

FiguRE 10. A homoclinic orbit

intersection induces that of infinitely many other homoclinic orbits: the $\operatorname{arc}$ of $W^{s}$ situated between $x$ and $\phi(x)$ crosses the unstable manifold $W^{u}$ infinitely often.

In [68] Shil'nikov wonders why Poincaré does not attach more importance to Hadamard's article, where one may obviously detect the presence of homoclinic orbits: an infinite sequence of 1,2,3 without repetitions and taking the value 1 only finitely many times defines a geodesic on the pants, which converges towards the periodic orbit $g_{1}$ both in the future and in the past. But Poincaré thinks that [60]:

The three-body problem should not be compared to the geodesics on surfaces of opposite curvatures; on the opposite, it should be compared to the geodesics on convex surfaces.

The opposition hyperbolic/elliptic, negative/positive curvature is not new.

The next step in the analysis of homoclinic orbits is due to George Birkhoff in 1935: through a cute geometrical argument, he establishes that one can find periodic points arbitrarily close to a transverse homoclinic point [13].

Then comes Smale's rediscovery 25 years later. If one considers a rectangle $R$ near the stable manifold, containing both the origin and the homoclinic point, like in Figure 11, then a sufficient iterate $\phi^{l}$ on $R$ acts like a horseshoe. One thus

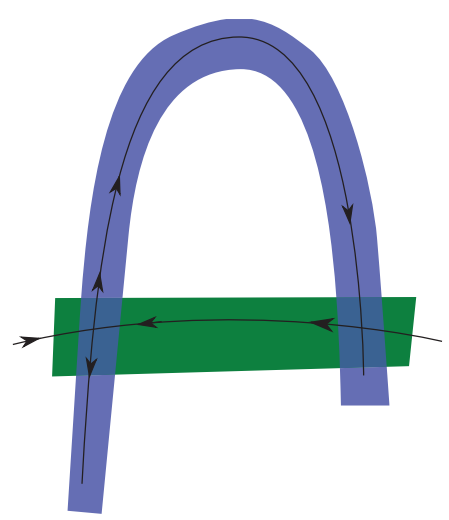

FiguRe 11. A homoclinic point and its horseshoe 
obtains a description of the "internal mechanism" of this thin tangle, for instance using sequences of symbols 0,1 as we have seen above. The presence of infinitely many periodic orbits becomes obvious. One can read in [68] an account on the evolution of the ideas around these homoclinic orbits.

\section{The Lorenz attractor}

How could one explain the lack of communication in the 1960s and 1970s between a theoretical physicist like Lorenz and a mathematician like Smale? The first one was working on the East Coast and the second one on the West Coast of the United States ... According to Williams [82], one reason would be the journal where Lorenz published his article ${ }^{12}$ :

Though many scientists, especially experimentalists, knew this article, it is not too surprising that most mathematicians did not, considering for example where it was published. Thus, when Ruelle-Takens proposed (1971) specifically that turbulence was likely an instance of a "strange attractor", they did so without specific solutions of the Navier-Stokes equations, or truncated ones, in mind. This proposal, controversial at first, has gained much favor.

It seems that Smale had very few physical motivations when cooking up his theory of hyperbolic systems, while physics itself does not seem to encompass many hyperbolic systems. This is at least Anosov's point of view [4]:

One gets the impression that the Lord God would prefer to weaken hyperbolicity a bit rather than deal with restrictions on the topology of an attractor that arise when it really is "1960s-model" hyperbolic.

Even nowadays, it is not easy to find physical phenomena with strictly hyperbolic dynamics (see however $[35,39]$ ). In my view, one of the main challenges of this part of mathematics is to "restore contact" with physics.

\subsection{Lorenz and his butterfly}

Lorenz's 1963 article [44] is magnificent. Lorenz had been studying for a few years simplified models describing the motion of the atmosphere, in terms of ordinary differential equations depending on few variables. For instance, in 1960 he describes a system he can explicitly solve using elliptic functions: the solutions are "still" quasiperiodic in time [42]. His 1962 article analyzes a differential equation in a space of dimension 12, in which he numerically detects a sensitive dependence to initial conditions [43]. But it is the 1963 paper which - for good reasons - lead him to fame. The aim of the paper is clear:

In this study we shall work with systems of deterministic equations which are idealizations of hydrodynamical systems.

\footnotetext{
${ }^{12}$ One may also wonder whether the prestigious journal where Williams published his paper [82] is accessible to physicists.
} 
After all, the atmosphere is made of finitely many particles, so one indeed needs to solve an ordinary differential equation in a space of "huge" dimension. But of course, such equations are "highly intractable", and one must treat them through partial differential equations; in turn, the latter must be discretized on a finite grid, leading to new ordinary differential equations depending on fewer variables, and probably more useful than the original ones. Lorenz discusses the type of differential equations he wants to study.

In seeking the ultimate behavior of a system, the use of conservative equations is unsatisfactory [...]. This difficulty may be obviated by including the dissipative processes, thereby making the equations non conservative, and also including external mechanical or thermal forcing, thus preventing the system from ultimately reaching a state at rest.

A typical differential equation presenting both viscosity and forcing has the following form:

$$
\frac{d x_{i}}{d t}=\sum_{j, k} a_{i j k} x_{j} x_{k}-\sum_{j} b_{i j} x_{j}+c_{i}
$$

where $\sum a_{i j k} x_{i} x_{j} x_{k}$ vanishes identically ${ }^{13}$ and $\sum b_{i j} x_{i} x_{j}$ is positive definite. The quadratic terms $a_{i j k} x_{j} x_{k}$ represent the advection, the linear terms $\sum b_{i j} x_{j}$ correspond to the friction and the constant terms to the forcing. Lorenz observes that under these conditions, the vector field is transverse to spheres of large radii (that is, of high energy), so that the trajectories entering a large ball will stay there forever. He can then discuss diverse notions of stability, familiar to contemporary mathematicians; periodicity, quasiperiodicity, stability in the sense of Poisson, etc. The bibliographic references in Lorenz's article include one article of Poincaré, but it is the famous one from 1881 [57]. In this article, founding the theory of dynamical systems, Poincaré introduces the limit cycles, and shows particular cases of the Bendixson-Poincaré theorem, introduces the first return maps etc., but there is no mention of chaotic behavior yet; chaos will be studied starting from the 1890 memoir we have discussed above, which Lorenz seems to have overlooked. Another bibliographic reference is a dynamical systems book by Birkhoff published in 1927. Again, this reference precedes Birkhoff's works in which he "almost" obtains a horseshoe ...

Then, Lorenz considers as example the phenomenon of convection. A thin layer of a viscous fluid is placed between two horizontal planes, set at two different temperatures, and one wants to describe the resulting motion. The high parts of the fluid are colder, therefore denser; they have thus a tendency to go down due to gravity, and are then heated when they reach the lower regions. The resulting circulation of the fluid is complex. Physicists know well the Bénard and Rayleigh experiments. Assuming the solutions are periodic in space, expanding in Fourier

\footnotetext{
${ }^{13}$ This condition expresses the fact that the "energy" $\sum x_{i}^{2}$ is invariant through the quadratic
} part of the field. 
series and truncating these series to keep only few terms, Salzman had just obtained an ordinary differential equation describing the evolution. Simplifying again this equation, Lorenz obtained "his" equation:

$$
\begin{aligned}
& d x / d t=-\sigma x+\sigma y \\
& d y / d t=-x z+r x-y \\
& d z / d t=x y \quad-b z .
\end{aligned}
$$

Here $x$ represents the intensity of the convection, $y$ represents the temperature difference between the ascending and descending currents, and $z$ is proportional to the "distortion of the vertical temperature profile from linearity, a positive value indicating that the strongest gradients occur near the boundaries". Obviously, one should not seek in this equation a faithful representation of the physical phenomenon ... The constant $\sigma$ is the Prandtl number. Guided by physical considerations, Lorenz is lead to choose the numerical values $r=28, \sigma=10, b=8 / 3$; it was a good choice, and these values remain traditional. He could then numerically solve these equations, and observe a few orbits. The electronic computer Royal McBee LGP-30 was rather primitive: according to Lorenz, it computed (only!) 1000 times faster than by hand ...

But Lorenz's observations are nevertheless remarkably fine. He first observes the famous sensitivity to initial conditions ${ }^{14}$. More importantly, he notices that these sensitive orbits still seem to accumulate on a complicated compact set, which is itself insensitive to initial conditions. He observes that this invariant compact set approximately resembles a surface presenting a "double" line along which two leaves meet each other.

Thus within the limits of accuracy of the printed values, the trajectory is confined to a pair of surfaces which appear to merge in the lower portion. [...] It would seem, then, that the two surfaces merely appear to merge, and remain distinct surfaces. [...] Continuing this process for another circuit, we see that there are really eight surfaces, etc., and we finally conclude that there is an infinite complex of surfaces, each extremely close to one or the other of the two merging surfaces.

Figure 12 is reprinted from Lorenz's article. Starting from an initial condition, the orbit rapidly approaches this "two-dimensional object" and then travels "on" this surface. The orbit then turns around the two holes, left or right, in a seemingly random way. Notice the analogy with Hadamard's geodesics turning around the bull's horns.

\footnotetext{
${ }^{14}$ The anecdote is quite well known "I started the computer again and went out for a cup of coffee"... It was told in the conference Lorenz gave on the occasion of the 1991 Kyoto prize, "A scientist by choice", which contains many other interesting things. In particular, he discusses there his relations with mathematics. In 1938 Lorenz is a graduate student in Harvard and works under the guidance of G. Birkhoff "on a problem in mathematical physics". He does not mention any influence of Birkhoff on his conception of chaos. A missed encounter? On the other hand, Lorenz mentions that Birkhoff "was noted for having formulated a theory of aesthetics". Almost all Lorenz's works, including a few unpublished ones, can be downloaded on http://eapsweb.mit.edu/research/Lorenz/publications.htm.
} 


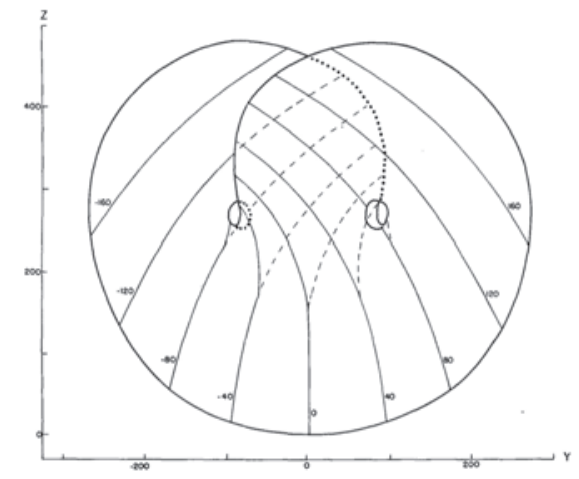

FiguRE 12. Lorenz's diagram

Besides, Lorenz studies the way the orbits come back to the "branching line" between the two leaves, which can be parametrized by an interval [0,1]. Obviously, this interval is not very well defined, since the two leaves do not really come in contact, although they coincide "within the limits of accuracy of the printed values". Starting from a point on this interval, one can follow the future trajectory and observe its return onto the interval. For this first return map $[0,1] \rightarrow[0,1]$, each point has one image but two preimages. This corresponds to the fact that, to go back in time and describe the past trajectory of a point in $[0,1]$, one should be able to see two copies of the interval; these copies are undistinguishable on the figure, so that two different past orbits emanate from the "same point" of the interval. But of course, if there are two past orbits starting from "one" point, there are four, then eight, etc., which is what Lorenz expresses in the above quotation. Numerically, the first return map is featured on the left part of Figure 13. Working by analogy, Lorenz compares this application to the (much simpler) following one: $F(x)=2 x$ if $0 \leqslant x \leqslant 1 / 2$ and $F(x)=2-2 x$ if $1 / 2 \leqslant x \leqslant 1$ (right part of Figure 13). Nowadays the chaotic behavior of this "tent map" is well known, but this was much less classical in $1963 \ldots$ In particular, the periodic points of $F$ are exactly the rational numbers with odd denominators, which are dense in $[0,1]$. Lorenz does not hesitate to claim that the same property applies to the iterations of the "true" return map. The periodic orbits of the Lorenz attractor are "thus" dense. What an intuition!

There remains the question as to whether our results really apply to the atmosphere. One does not usually regard the atmosphere as either deterministic or finite, and the lack of periodicity is not a mathematical certainty, since the atmosphere has not been observed forever.

To summarize, this article contains the first example of a dissipative and physically relevant dynamical system presenting all the characteristics of chaos. The orbits are unstable but their asymptotic behavior seems relatively insensitive to initial 

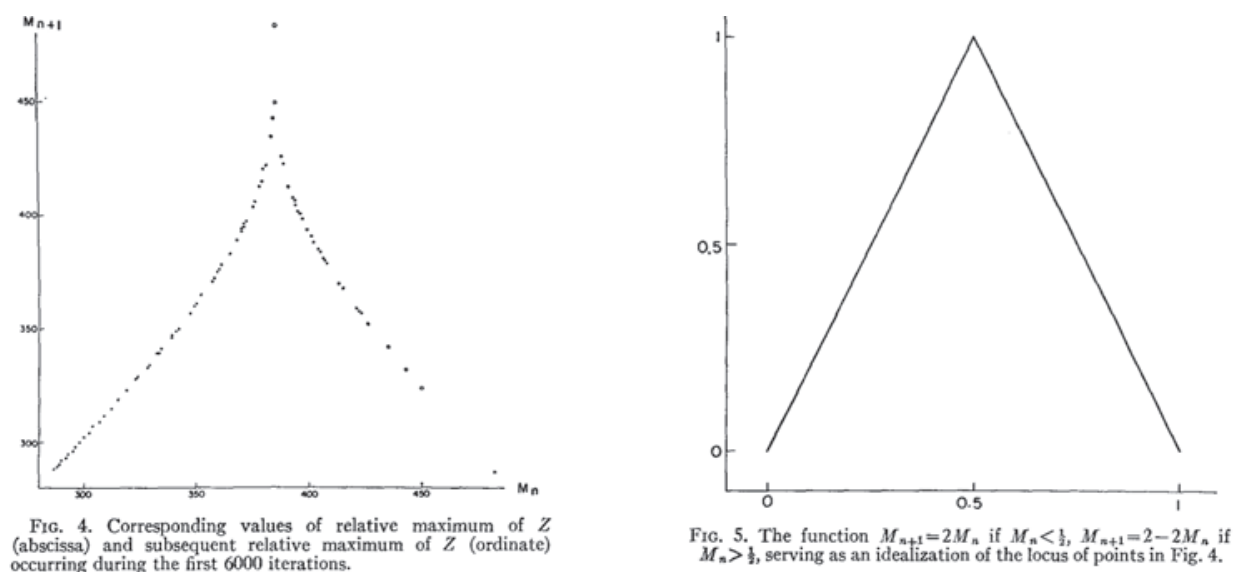

FiguRE 13. Lorenz's graphs of first return maps

conditions. None of the above assertions is justified, at least in the mathematical sense. How frustrating!

Very surprisingly, an important question is not addressed in Lorenz's article. The observed behavior happens to be robust: if one slightly perturbs the differential equation, for instance by modifying the values of the parameters, or by adding small terms, then the new differential equation will feature the same type of attractor with the general aspect of a surface. This property will be rigorously established later, as we will see.

Everybody has heard of the "butterfly effect". The terminology seems to have appeared in the best-selling book of Gleick [29], and be inspired by the title of a conference by Lorenz in 1972 [45].

\subsection{Guckenheimer, Williams and their template}

The Lorenz equation pops up in mathematics in the middle of the 1970s. According to Guckenheimer [32], Yorke mentioned to Smale and his students the existence of this equation, which was not encompassed by their studies. The well-known 1971 article by Ruelle and Takens on turbulence [67] still proposes hyperbolic attractors as models, but in 1975 Ruelle observes that "Lorenz's work was unfortunately overlooked" [65]. Guckenheimer and Lanford are among the first people to show some interest in this equation (from a mathematical point of view) [31, 40]. Then the object will be fast appropriated by mathematicians, and it is impossible to give an exhaustive account of all their works. As soon as 1982 a whole book is devoted to the Lorenz equation, although it mostly consists in a list of open problems for mathematicians [79].

I will only present here the fundamental works of Guckhenheimer and Williams, who constructed the geometric Lorenz models [33, 82] (independently 
from Afraimovich, Bykov and Shil'nikov [2]). The initial problem consists in justifying the phenomena observed by Lorenz on his equation. We have seen that this equation is itself a rough approximation of the physical phenomenon. Proving that the precise Lorenz equation satisfies the observed properties is thus not the most interesting issue. Guckenheimer and Williams have another aim: they consider the behaviors observed by Lorenz as an inspiration, in order to construct vector fields, called geometric Lorenz models, satisfying the following properties:

- for each or these fields, the set of nonwandering points is not hyperbolic, since it contains both nonsingular points and a singular one

- the fields are not structurally stable;

- the fields form an open set in the space of vector fields.

Let us first consider a linear vector field:

$$
\frac{d x}{d t}=a x, \quad \frac{d y}{d t}=-b y, \quad \frac{d z}{d t}=-c z
$$

with $0<c<a<b$. Let $C$ be the square $[-1 / 2,1 / 2] \times[-1 / 2,1 / 2] \times\{1\} \subset \mathbb{R}^{3}$. The orbit starting from a point $\left(x_{0}, y_{0}, 1\right)$ in this square is $\left(x_{0} \exp (a t), y_{0} \exp (-b t)\right.$, $\exp (-c t))$. Call $T_{ \pm}$the triangular zones where these orbits intersect the planes given by $\{x= \pm 1\}$. They are defined by the equations $x= \pm 1,|y| \leqslant \frac{1}{2}|z|^{b / c}$ and $z>0$, so they are "triangles" with their lower corner being a cusp. One then considers the zone ("box") $B$ swept by the orbits starting from $C$ until they reach $T_{ \pm}$, to which one adds the future orbits of the points in $\{x=0 ;-1 / 2 \leqslant y \leqslant$ $1 / 2 ; z=1\}$ (which never intersect $T_{ \pm}$), as well as the wedge $\{-1 \leqslant x \leqslant 1 ; y=$ $0 ; z=0\}$ (see Figure 14).

The "Lorenz vector field" we will construct coincides with this linear vector field inside the box $B$. Outside one proceeds such that the orbits exiting from $T_{ \pm}$

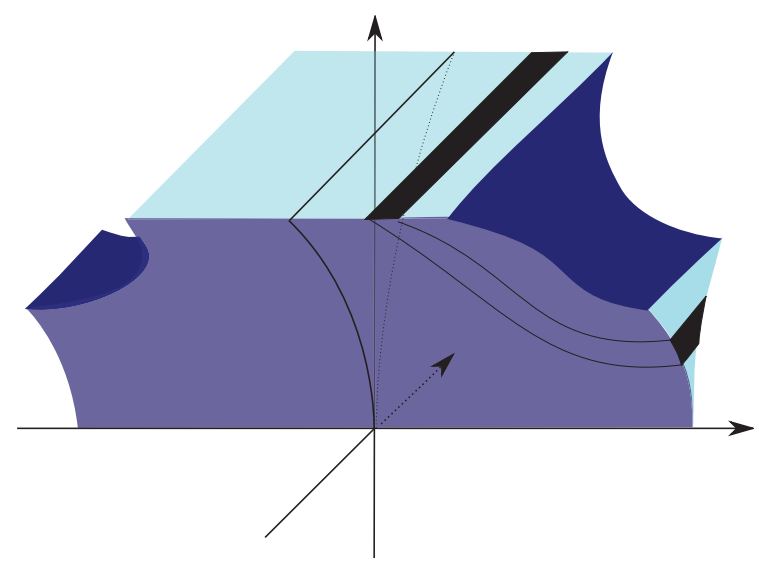

Figure 14. The box $B$ 


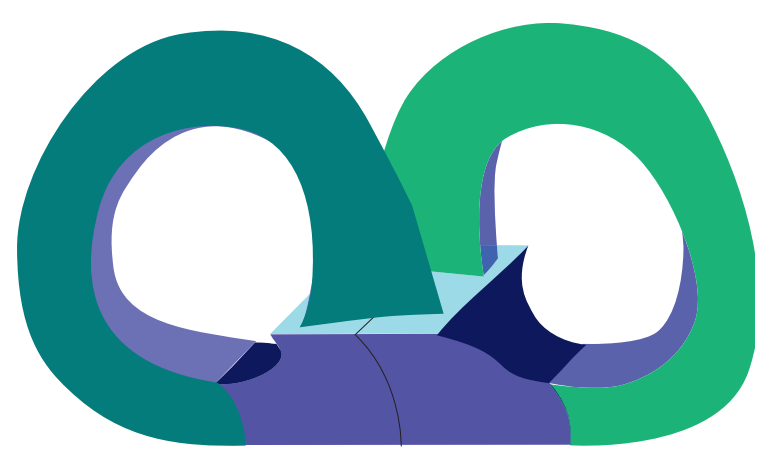

Figure 15. A Lorenz geometric model

come back inside the square $C$. One then obtains a vector field defined only inside a certain domain $D$ sketched in Figure 15 .

The main objective is to understand the dynamics inside $D$, but one can also extend the vector field outside this domain to get a globally defined field. Such a vector field $X$ is a geometric Lorenz model.
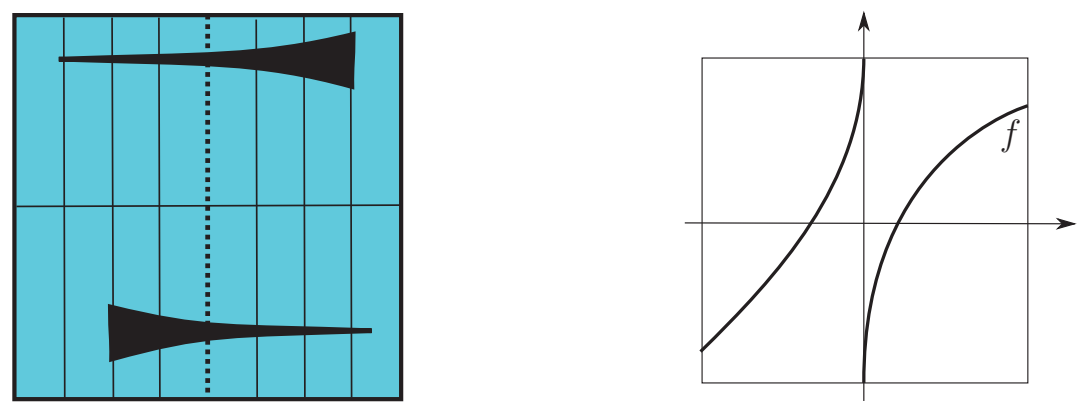

FiguRE 16. Return maps

An important remark is that not all the points of the triangles originate from the square: the tips do not, since they come from the singular point at the origin. There are several ways to organize the return from $T_{ \pm}$onto $C$; one can make sure that the Poincaré return map $F: C \rightarrow C$ has the following form:

$$
F(x, y)=(f(x), H(x, y)) .
$$

Technically, one requires that $H(x, y)>1 / 4$ for $x>0$ and $H(x, y)<1 / 4$ for $x<0$. Furthermore, that the map $f:[-1 / 2,1 / 2] \rightarrow[-1 / 2,1 / 2]$ satisfies the following conditions:

1. $f\left(0^{-}\right)=1 / 2, f\left(0^{+}\right)=-1 / 2$;

2. $f^{\prime}(x)>\sqrt{2}$ for all $x$ in $[-1 / 2,1 / 2]$. 
The second conditions implies that for all interval $J$ contained in $[-1 / 2,1 / 2]$, there exists an integer $l>0$ such that $f^{l}(J)=[-1 / 2,1 / 2]^{15}$

To describe the structure of the orbits inside the box, Williams introduces the concept of template. Figure 17 is reprinted from $[15]^{16}$ : We are dealing with a

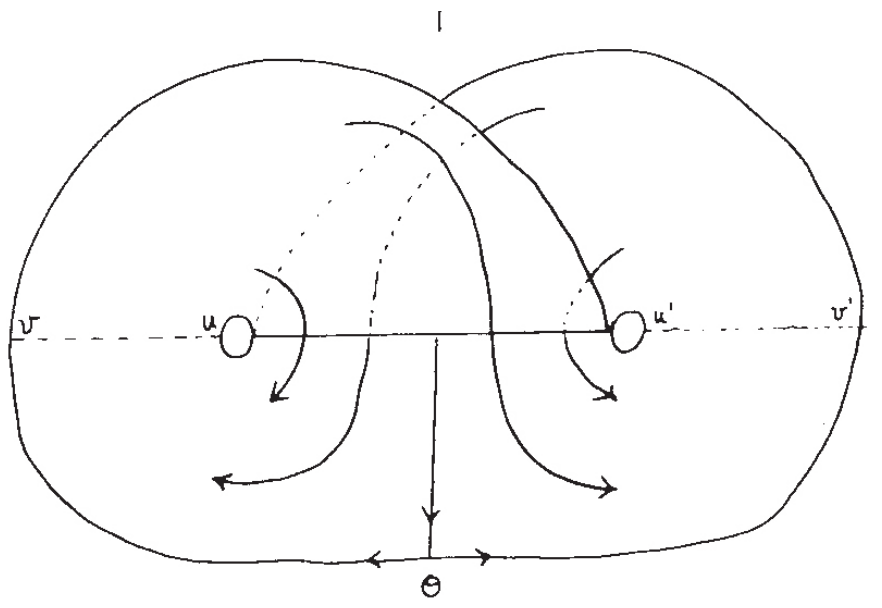

FiguRe 17. The template

branched surface $\Sigma \subset \mathbb{R}^{3}$ embedded in space, on which one can define a semiflow $\psi^{t}(t \geqslant 0)$. A semiflow means that $\psi^{t}: \Sigma \rightarrow \Sigma$ is defined only for $t \geqslant 0$ and that $\psi^{t_{1}+t_{2}}=\psi^{t_{1}} \circ \psi^{t_{2}}$ for all $t_{1}, t_{2}$. The trajectories of the semiflow are sketched on the figure: a point in $\Sigma$ has a future but has no past, precisely because of the two leaves which meet along an interval. The first return map on this interval is chosen to be the map $f$ defined above. The dynamics of the semiflow is easy to understand: the orbits turn on the surface, either on the left or on the right wing, according to the signs of the iterates $f^{k}(x)$

We shall now construct a flow starting from the semiflow using a well-known method, the projective limit. One considers the abstract space $\hat{\Sigma}$ of the curves $c: \mathbb{R} \rightarrow \Sigma$ which are trajectories of $\psi^{t}$ in the following sense: for all $s \in \mathbb{R}$ and $t \in \mathbb{R}^{+}$, one has $\phi^{t}(c(s))=c(s+t)$. Given a point $x$ on $\Sigma$, to choose such a curve $c$ with $c(0)=x$ amounts to "selecting a past" for $x$ : on goes backwards in time along the semiflow, and at each crossing of the interval, one chooses one of the two possible preimages. The map $c \in \hat{\Sigma} \mapsto c(0) \in \Sigma$ thus has totally discontinuous fibers, which are Cantor sets. The space $\hat{\Sigma}$ is an abstract compact set equipped with a flow $\hat{\psi}^{t}$ defined by $\hat{\psi}^{t}(c)(s)=c(s+t)$, which now makes sense for all $t \in \mathbb{R}$.

\footnotetext{
${ }^{15}$ The fact that the graph of $f$ does not resemble Figure 13 is due to a different choice of notations. ${ }^{16}$ Incidentally, this figure shows that the quality of an article does not depend on that of its illustrations ...
} 
Let us fix a Lorenz model $X$ generating a flow $\phi^{t}$ and associated with a first return map $f$. Williams shows in [82] that: There exists a compact $\Lambda$ contained in the box D, such that:

- the $\omega$-limit set of each point in $D$ is contained in $\Lambda$;

- $\Lambda$ is invariant through $\phi^{t}$, and the restriction of $\phi^{t}$ on $\Lambda$ is topologically conjugate with $\hat{\psi}^{t}$ acting on $\hat{\Sigma}$;

- $\Lambda$ is topologically transitive: it contains an orbit which is dense in $\Lambda$;

- the union of all periodic orbits is dense in $\Lambda$.

We have now justified Lorenz's intuition, according to which the attractor $\Lambda$ behaves as a surface "within the limits of accuracy of the printed values".

We also see that the topological dynamics of the vector field is completely determined by that of the map $f$. To understand it, one uses the notion of kneading sequence introduced around the same time in a more general context. Take the two sequences $\alpha_{k}, \beta_{k}= \pm$ given by the signs of $f^{k}\left(0^{-}\right)$et $f^{k}\left(0^{+}\right)$for $k \geqslant 0$ (the sign of 0 is defined to be + ). Obviously, two applications $f$ of the above type which are conjugated by a homeomorphism (preserving the orientation) define the same sequences $\alpha_{k}, \beta_{k}$, and the converse is true. Back to the vector field, these two sequences can be obtained by considering the two unstable separatrices starting from the origin, which will intersect the square $C$ infinitely many times, either in the part $x>0$ or in the part $x<0$. The two sequences precisely describe the forward evolution of these two separatrices. It should be clear that these fields are structurally unstable, since a slight perturbation can change the sequences $\alpha_{k}, \beta_{k}$.

Guckenheimer and Williams prove that the two sequences contain all the topological information on the vector field. Precisely, the establish the following theorem:

There exists an open $\mathcal{U}$ in the space of vector fields in $\mathbb{R}^{3}$ and a continuous map $\pi$ from $\mathcal{U}$ into a two-dimensional disk, such that two fields $X, Y$ on $\mathcal{U}$ are conjugate through a homeomorphism close to the identity if and only if $\pi(X)=\pi(Y)$.

We won't dwell on the proofs, but will insist on an important point: the Lorenz flow, although nonhyperbolic, is still "singular hyperbolic". Namely, at each point $x$ of the attractor $\Lambda$, one can decompose the tangent space into a direct sum of a line $E_{x}^{s}$ and a plane $F_{x}$, such that the following properties hold:

- $E_{x}^{s}$ and $F_{x}$ depend continuously of the point $x \in \Lambda$, and are invariants through the differential of the flow $\phi^{t}$;

- the vectors in $E_{x}^{s}$ are contracted by the flow: there exists $C>0, \lambda>0$ such that for all $t>0$ and all $v \in E_{x}^{s}$, one has $\left\|d \phi^{t}(v)\right\| \leqslant C \exp (-\lambda t)\|v\|$;

- the vectors in $F_{x}$ are not necessarily expanded by the flow, but they cannot be contracted as much as the vectors in $E^{s}$. Precisely, if $u \in F_{x}$ and $v \in E_{x}^{s}$ are unitary, one has $\left\|d \phi^{t}(u)\right\| \geqslant C^{-1} \exp (\lambda t)\left\|d \phi^{t}(v)\right\|$ for all $t>0$;

- the flow uniformly expands the two-dimensional volume along

$$
F_{x}: \operatorname{det}\left(d \phi^{t} \mid F_{x}\right) \geqslant \exp \left(\lambda^{\prime} t\right)
$$

for some $\lambda^{\prime}>0$ and all $t>0$. 
For each $x \in \Lambda$ one may consider the set $W^{s}(x)$ of the points $y \in \mathbb{R}^{3}$ such that the distance $\left\|\phi^{t}(x)-\phi^{t}(y)\right\|$ goes to zero faster than $\exp (-\lambda t)$. It is a smooth curve with tangent in $x$ given by $E_{x}^{s}$. The collection of these curves defines a foliation of an open neighborhood of $\Lambda$, justifying the terminology "attractor": all the points in this neighborhood are attracted by $\Lambda$ and their trajectories are asymptotes of trajectories in $\Lambda$. The branched surface $\Sigma$ is constructed from the local leaves of this foliation. Of course, one needs to show that all these structures exist, and that they persist upon a perturbation of the vector field.

Hence, the open set in the space of vector fields detected by Guckenheimer and Williams trespasses the hyperbolic systems, but the dynamics of this type of fields can still be understood (at list qualitatively). The specificity of these fields is that they are, in some sense, suspensions of maps $f$ on the interval, but also contain a singular point in their nonwandering set. In some sense, their dynamics can be translated into a discrete time dynamics, but the presence of the singular point shows that the first return time on $C$ is unbounded. It would be too naive to believe that this type of phenomenon, together with hyperbolic systems, suffice to understand "generic dynamics". We will see later some other types of phenomena (different from hyperbolicity) happening in a stable manner. But the "Lorenz phenomenon" I have just described is doubtlessly one of the few phenomena representing a generic situation. We will come back later to this question.

As we have mentioned, the geometric models for the Lorenz attractor have been inspired by the original Lorenz equation, but it wasn't clear whether the Lorenz equation indeed behaves like a geometric model. This question was not really crucial, since Lorenz could clearly have made other choices to cook up his equation, which resulted from somewhat arbitrary truncations of Fourier series. Lorenz himself never claimed that his equation had any physical sense. Nevertheless, the question of the connection between the Lorenz equation and the GuckenheimerWilliams dynamics was natural, and Smale chose it as one of the "mathematical problems for the next century" in 1998 [78]. The problem was positively solved by Tucker [80] (before the "next century"!). The goal was to construct a square $C$ adapted to the original Lorenz equation, the first return map on $C$, and to check that they have the properties required by the geometric model. The proof uses a computer, and one needs to bound from above the errors. The major difficulty which makes the problem quite delicate - is due to the presence of the singular point, and the fact that the return time may become very large ... For a brief description of the method used by Tucker, see for instance [81].

\section{The topology of the Lorenz attractor}

\subsection{Birman, Williams and their "can of worms"}

We have seen that the periodic orbits of a geometric Lorenz model are dense in the attractor. To better understand the topology of the attractor, Birman and Williams had the idea to consider these periodic orbits as knots. A knot is a 
closed oriented curve embedded in space without double points. A topologist will consider that two knots are identical (the technical term is isotopic) if it is possible to continuously deform the former into the latter without any double point. Now the questions are: which knots are represented by at least one periodic orbit of the Lorenz model? Can a single knot be represented by infinitely many periodic orbits? Beyond knots, one can also consider the links, which are unions of finitely many disconnected knots. Each collection of finitely many periodic orbits defines a link. Since the periodic orbits are dense in the attractor, one can hope to approximate the latter by a link containing a huge number of periodic orbits. The article [15] nicely mixes topology and dynamics.

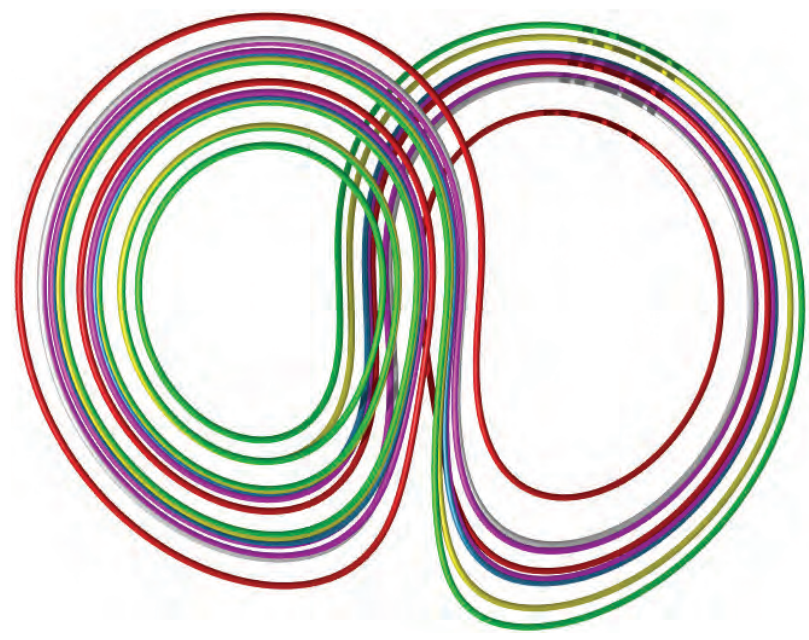

FiguRE 18. A few periodic orbits

A priori, this study of knots and links should be performed for each geometric Lorenz model, that is for each return map $f$, or more precisely for each choice of a pair of kneading sequences $\alpha, \beta$. However, we may restrict ourselves to the particular case of the "multiplication by two" case, by setting $f_{0}(x)=2 x+1$ for $x \in\left[-1 / 2,0\left[\right.\right.$ and $f_{0}(x)=2 x-1$ for $x \in[0,1 / 2]$. A point in $[-1 / 2,1 / 2]$ is fully determined by the sequence of signs of its iterates by $f_{0}$, and each sequence of signs corresponds to a point in $[-1 / 2,1 / 2]$. This is nothing but the dyadic decomposition of numbers in $[-1 / 2,1 / 2]$. If $f$ is the return map on $[-1 / 2,1 / 2]$ for a given geometric Lorenz model, one can associate to each point $x \in[-1 / 2,1 / 2]$ the unique point $h(x) \in[-1 / 2,1 / 2]$ such that for each $k \geqslant 0$ the numbers $f^{k}(x)$ and $f_{0}^{k}(h(x))$ have the same sign. This defines an injection $h:[-1 / 2,1 / 2] \rightarrow[-1 / 2,1 / 2]$ such that $h \circ f=f_{0} \circ h$, and one can thus think that $f_{0}$ "contains" all the onedimensional dynamics we are interested in. Of course, the map $h$ is not always a bijection, depending on the specific geometric Lorenz model. We will study the maximal case of $f_{0}$, since it contains all the others. Strictly speaking, $f_{0}$ cannot be 
a first return map of a geometric Lorenz model, since its derivative does not blow up near $x=0$. However, starting from $f_{0}$ one may construct, like in the previous section, a topological semiflow on the branched surface, and then a flow through a projective limit. This topological flow is perfectly adapted to our problem, which is the nature of knots and links formed by the periodic orbits of the geometric Lorenz models.

Each periodic orbit of the Lorenz flow can be projected onto the template, so it is associated with a periodic orbit of $f$. One could fear that this projection could modify the topology of the knot through the appearance of double points. But this does not happen because the projection on the template occurs along the (one-dimensional) stable manifolds, and clearly a stable manifold can meet a periodic orbit on at most one point (two different points cannot simultaneously be periodic and asymptotes of each other).

The periodic points of $f_{0}$ are easy to determine: they are the rational numbers with denominators of the form $2(2 l+1)$, with $l \in \mathbb{Z}$. If $x$ is such a periodic point of period $k$, one constructs a braid as follows: inside the square $[-1 / 2,1 / 2] \times[0,1]$, for $i=0, \ldots, k-1$ one connects the points $\left(f^{i}(x), 0\right)$ and $\left(f^{i+1}(x), 1\right)$ by a segment, such that the segments "climbing to the right" are below those "climbing to the left", like on Figure 19. Then, one closes the braid as usual in topology, to obtain

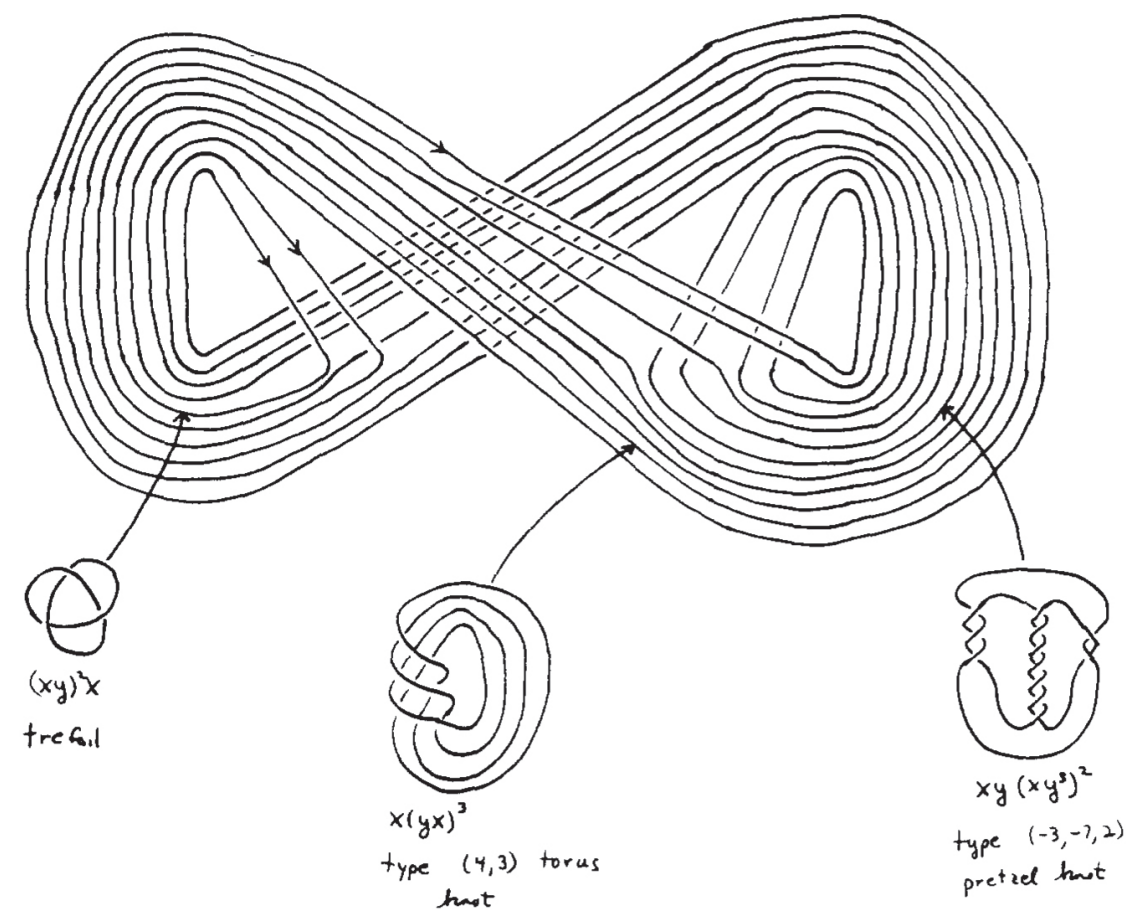

Figure 19. A Lorenz link 
a knot $k(x)$. Starting from a finite number of periodic points $x_{1}, x_{2}, \ldots, x_{l}$, one obtains a link. Those are called the "Lorenz knots and links".

Before describing some of the results obtained in [15, 83], I should remind some definitions related with knots. An oriented knot is always the boundary of a oriented surface embedded in the three-dimensional space (called its Seifert surface). The minimal genus of such a surface is the genus of the knot. If a knot cannot be obtained as the connected sum of two nontrivial knots, it is said to be prime. A knot is said to be chiral if it cannot be continuously deformed into its mirror image. Given a knot $k$ embedded in the three-dimensional sphere $\mathbb{S}^{3}$ (union of $\mathbb{R}^{3}$ and a point at infinity), it is said to be fibered if the complement $\mathbb{S}^{3} \backslash k$ fibres on the circle; this means that there exists a family of surfaces with boundaries, parametrized by an angle $\theta \in \mathbb{S}^{1}$, which all share the same boundary $k$ but do not intersect each other outside their boundary, and which altogether cover the whole sphere. Near the knot, the surfaces looks like the pages of a book near the binding. Finally, given two disjoint knots $k_{1}$ and $k_{2}$, one can define as follows their linking number: choose an oriented surface with boundary $k_{1}$, and count the algebraic intersection number of $k_{2}$ with this surface. This integer enl $\left(k_{1}, k_{2}\right)$ is independent of the chosen surface, and $\operatorname{enl}\left(k_{1}, k_{2}\right)=\operatorname{enl}\left(k_{2}, k_{1}\right)$.

Here are a few properties of the Lorenz links:

- The genus of a Lorenz knot can be arbitrary large.

- The linking number of two Lorenz knots is always positive.

- The Lorenz knots are prime.

- The Lorenz links are fibered.

- The nontrivial Lorenz knots are chiral.

The Lorenz knots are very particular. For instance, using a computer and the tables containing the 1701936 prime knots representable by plane diagrams with less than 16 crossings, one can show that only 21 of them are Lorenz knots [28]. More information on the Lorenz knots and the recent developments on the subject can be found in $[22,14]$. Surprisingly, Ghrist has shown that if one embeds the branched surface in 3-space by twisting one of the wings by a half-turn, the resulting flow is universal: all the links are represented by (finite collections of) periodic orbits of that flow [25].

\subsection{The right-handed attractor...}

Try to see the attractor as a "topological limit" of its periodic orbits, and take into account the fact that each finite union of periodic orbits defines a fibered link; how do these circle fibrations behave when the number of components of the link tends to infinity? In a way, one would like to see the complement of the attractor itself as a fibered object ... In [27] I propose a global description of these fibrations, based on the concept of right-handed vector field.

Consider a nonsingular vector field $X$ on the sphere $\mathbb{S}^{3}$, generating a flow $\phi^{t}$. Let us call $\mathcal{P}$ the convex compact set formed by the probability measures invariant by the flow. This space contains, for instance, the probability measures 
equidistributed on the periodic orbits (if any), as well as their convex combinations. A general invariant probability measure can be seen as a generalized periodic orbit (a foliated cycle in Sullivan's terminology). We have seen that two knots in the sphere always define a linking number; I show that one can also define a linking quadratic form enl : $\mathcal{P} \times \mathcal{P} \rightarrow \mathbb{R}$. The flow is then called right-handed if enl only takes positive values. For the Lorenz flow, enl is only nonnegative, but it is positive when restricted to invariant measures which do not charge the singular point. This fact can be directly "read" on Williams's template, since two arcs of trajectory only have positive intersections when projected onto the plane. The Lorenz flow is thus not strictly right-handed.

One of the main results in [27] is that a right-handed flow is always fibered, in the following sense. One can find a positive Gauss linking form on the flow. Precisely, there exists a $(1,1)$-differential form $\Omega$ on $\mathbb{S}^{3} \times \mathbb{S}^{3}$ minus the diagonal, such that:

- if $\gamma_{1}, \gamma_{2}: \mathbb{S}^{1} \rightarrow \mathbb{S}^{3}$ are two disjoint closed curves, their linking number is given by the Gauss integral $\iint \Omega_{\gamma_{1}\left(t_{1}\right), \gamma_{2}\left(t_{2}\right)}\left(\frac{d \gamma_{1}}{d t_{1}}, \frac{d \gamma_{2}}{d t_{2}}\right) d t_{1} d t_{2}$;

- if $x_{1}, x_{2}$ are two distinct points, then $\Omega_{x_{1}, x_{2}}\left(X\left(x_{1}\right), X\left(x_{2}\right)\right)>0$.

Interestingly, such a Gauss form directly provides a fibration on the complement of each periodic orbit. Indeed, for $\mu$ any invariant probability measure, the integral

$$
\omega_{x}(v)=\int \Omega_{x, y}(v, X(y)) d \mu(y)
$$

defines a closed and nonsingular 1-form on the complement of the support of $\mu$. If $\mu$ is supported on a periodic orbit, the form $\omega$ admits a multivalued primitive, which defines a fibration on the circle of the complement of the orbit.

Even though the Lorenz flow does not strictly belong to this frame, I show in [27] how this construction allows to better understand the results of Birman and Williams I have described above.

\subsection{From Lorenz back to Hadamard...}

Hadamard studies the geodesics on negatively curved pants, and shows that they can be described by the sequences of symbols enumerating the crossed seams. Williams analyzes the trajectories of the Lorenz attractor by a semiflow on the "Lorenz template", itself described by two sequences of symbols, following the wings (left or right) successively crossed by two limiting trajectories. It is thus not surprising to find a connection between these two dynamics. Such a connection was indeed exhibited in [26].

Following a nonwandering geodesic on the pants $P$, after each crossing with a seam one may consider to turn right or turn left to reach the next seam. It is thus possible to associate to each nonwandering geodesic a bi-infinite sequence of "left/right" symbols. Yet, this new coding is not perfect, because it is not bijective. Assume the pants is embedded symmetrically in space, meaning that it is invariant 


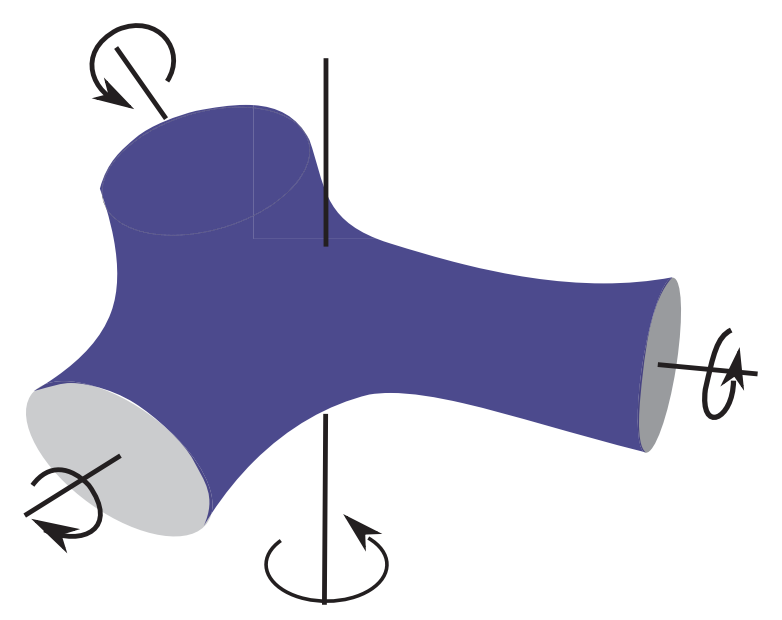

Figure 20. Symmetries of the pants

through six rotations (the identity, two rotations of order three, and three rotations of order two), as in Figure 20.

Obviously, each of these rotations maps a geodesic of $P$ into another geodesic, which shares the same "left-right" sequence. One is thus brought to consider the quotient $\hat{P}$ of $P$ by this group of order six. This quotient is not exactly a surface, due to the intersection points of the rotation axes with $P$. Ignoring this "detail", the geodesics of $\hat{P}$ are bijectively coded by the sequences "left-right", like the orbits of the Lorenz flow.

We now recognize in $\hat{P}$ a variant of the modular surface, quotient of the Poincaré half-plane $\mathbb{H}=\{z \in \mathbb{C} \mid \Im z>0\}$ through the action of $\Gamma=\operatorname{PSL}(2, \mathbb{Z})$ by Moebius transformations. The subgroup $\Gamma[2]$ formed by the matrices congruent to the identity modulo 2 , acts without fixed points on $\mathbb{H}$, and the quotient is a sphere minus three points. The group $\Gamma / \Gamma[2]$ of order six acts on this punctured sphere similarly as it acts on $P$. A geodesic of the modular surface is thus coded by a sequence of "left-right" symbols, like an orbit of the Lorenz flow.

To go further, one should compare the spaces supporting these two dynamics. For the Lorenz equation, it is the usual space $\mathbb{R}^{3}$. For the modular geodesic flow, it is the quotient $\operatorname{PSL}(2, \mathbb{R}) / \operatorname{PSL}(2, \mathbb{Z})$ : this is due to the fact that $\operatorname{PSL}(2, \mathbb{R})$ can be identified with the unitary tangent bundle to $\mathbb{H}$. Figure 21 displays a fundamental domain for the action of $\operatorname{PSL}(2, \mathbb{Z})$ on the Poincaré half-plane. Three copies of this domain form a circular triangle, with corners sitting on the real axis. Two copies of this triangle form a fundamental domain for $\Gamma[2]$.

In [26] I use the topological identification (through the classical modular forms $)$ of $\operatorname{PSL}(2, \mathbb{R}) / \operatorname{PSL}(2, \mathbb{Z})$ with the complement in the sphere $\mathbb{S}^{3}$ of the trefoil knot, in order to represent the modular geodesics on the sphere. I can then "identify" the topological dynamics of the Lorenz flow and the modular geodesic 


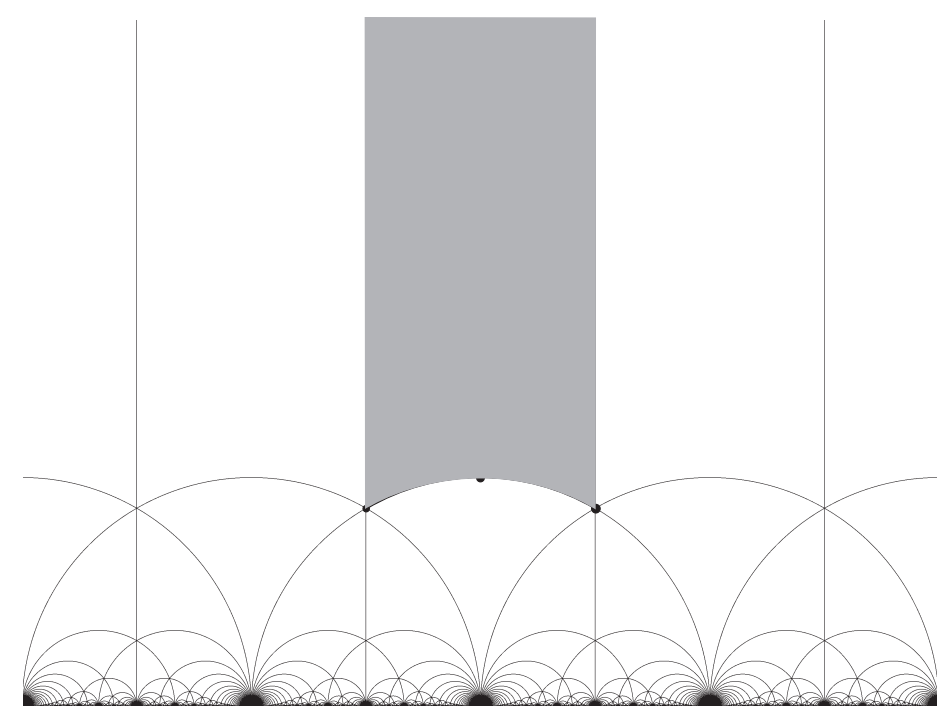

Figure 21. A fundamental domain for the action of $\operatorname{PSL}(2, \mathbb{Z})$ on the half-plane

flow. For instance, I show that the periodic geodesics on the modular surface, seen as knots on the sphere, can be deformed into the Lorenz knots. The left part of Figure 22 shows one of the periodic orbits of the modular geodesic flow, embedded in the complement of (a version of) the Lorenz template. The right part shows the Lorenz orbit with the same symbolic coding. The central part illustrates a step in the deformation. For more explanations, see [26, 28].
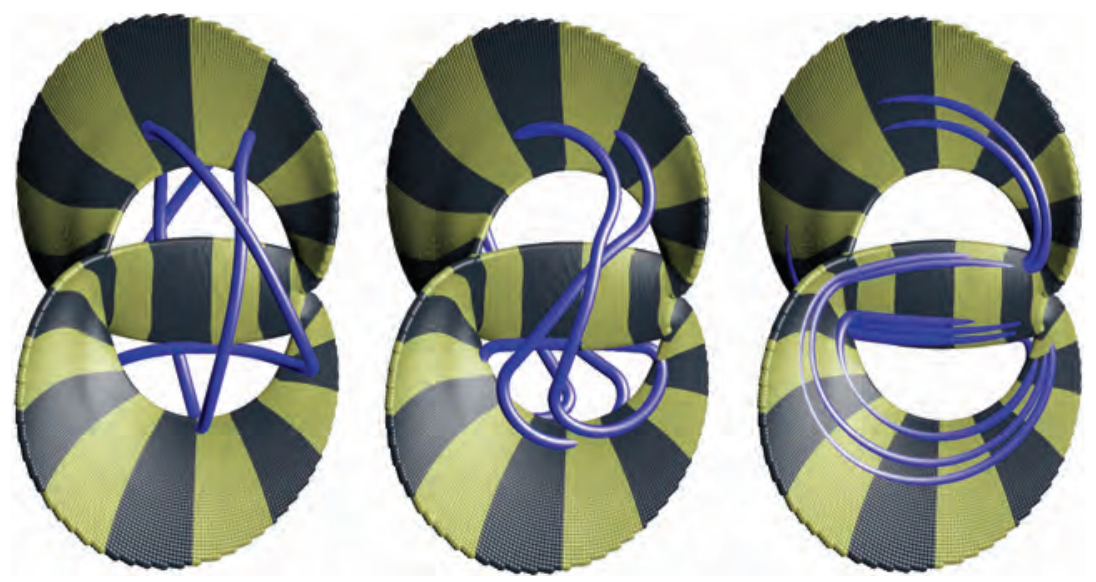

FIGURE 22. Deformation of a modular knot into a Lorenz knot 
The analogy between a fluid motion and a geodesic flow is not new. In a remarkable 1966 article, Arnold showed that the Euler equation for perfect fluids is nothing but the equation for the geodesics on the infinite-dimensional group of volume preserving diffeomorphisms [7]. Besides, he shows that the sectional curvatures are "often" negative, inducing him to propose a behavior à la Hadamard for the solutions of the Euler equation. Of course, Lorenz does not consider perfect fluids, he starts from the Navier-Stokes equation instead of the Euler equation; still, the motivations are similar.

\section{The attractor as a statistical object}

\subsection{Physical measures}

As we have seen, one of the major ideas to "escape" the sensitivity to initial conditions is to introduce probabilities.

Lorenz is one of the people who have most clearly expressed this idea: "over the years minuscule disturbances neither increase nor decrease the frequency of occurrence of various weather events such as tornados; the most they may do is to modify the sequence in which these events occur."

A flow $\phi^{t}$ on a compact manifold $V$ preserves at least one probability measure. This results, for instance, from the fact that this flow acts on the convex compact set of probability measures on $V$ (in an affine way); such an action of an abelian group always admits a fixed point. This mathematical existence theorem will certainly not impress any physicist! But, once we have "selected" an invariant probability measure $\mu$, we may invoke Birkhoff's ergodic theorem: for each function $u: V \rightarrow \mathbb{R}$ integrable w.r.t. the measure $\mu$, the asymptotic time average

$$
\tilde{u}(x)=\lim _{T \rightarrow+\infty} \frac{1}{T} \int_{0}^{T} u\left(\phi^{t}(x)\right) d t
$$

exists for $\mu$-almost all point $x$. The function $\tilde{u}$, defined almost everywhere, is obviously constant along the orbits of the flow. One can choose ${ }^{17}$ the measure $\mu$ to be ergodic, so that any invariant function is constant almost everywhere. This ergodic theorem could be considered as fulfilling Lorenz's wish: the frequency of an event does not depend on the initial conditions. However, the difficulty lies in the fact that a given flow $\phi^{t}$ usually preserves infinitely many measures, and the sets of full measure for two invariant measures $\mu_{1}$ and $\mu_{2}$ may be disjoint. The question is to determine whether there exists a "physically meaningful" invariant measure.

In the early 1970s, Sinai, Ruelle and Bowen have discovered a fundamental concept to answer this question $[71,66,17]$.

\footnotetext{
${ }^{17}$ An invariant probability measure is ergodic if any invariant subset has measure zero or one. The ergodic measures are the extremal points of the convex compact set of invariant probability measures, showing that there exist such measures (this existence proof is rather abstract).
} 
Let us recall that on a manifold there is no intrinsic notion of Lebesgue measure, yet the Lebesgue negligible sets are well defined, that is, they do not depend on the choice of coordinate system. For this reason, we may say that a measure is absolutely continuous with respect to "the" Lebesgue measure. Lebesgue negligible sets could be considered to be negligible for a physicist as well...

It is time to give a precise definition of the word attractor I have used many times. A compact set $\Lambda$ in $V$ is an attractor if:

- $\Lambda$ is invariant by the flow $\phi^{t}$;

- there exists a point in $\Lambda$ the orbit of which is dense in $\Lambda$;

- the basin of attraction $B(\Lambda)=\{x \in V \mid \omega(x) \subset \Lambda\}$ has nonzero Lebesgue measure.

A probability measure $\mu$ invariant by $\phi^{t}$ is an SRB measure (for Sinai-RuelleBowen) if, for each continuous function $u: V \rightarrow \mathbb{R}$, the set of points $x$ such that

$$
\lim _{T \rightarrow+\infty} \frac{1}{T} \int_{0}^{T} u\left(\phi^{t}(x)\right) d t=\int u d \mu
$$

has nonzero Lebesgue measure.

This set of points is called the basin of $\mu$, and denoted by $B(\mu)$.

Of course there are similar definitions for discrete time dynamical systems $\phi: V \rightarrow V$ (not necessarily bijective).

A simple example, yet not generic in our context, is that of a flow preserving a probability measure absolutely continuous w.r.t. the Lebesgue measure, and which is ergodic. Indeed, in that case the ergodic theorem precisely means that the time averages converge almost surely towards the spatial average. But I have already mentioned that our discussion mostly concerns the dissipative dynamics... An SRB measure is an invariant measure which "best remembers the Lebesgue measure".

Before giving more examples, let us start by a counterexample, due to Bowen. Let us consider a vector field admitting two saddle connections, as on Figure 23. For an initial condition $x$ inside the region bounded by the separatrices, the future orbit will accumulate on the two singular points. Since the dynamics is slow near

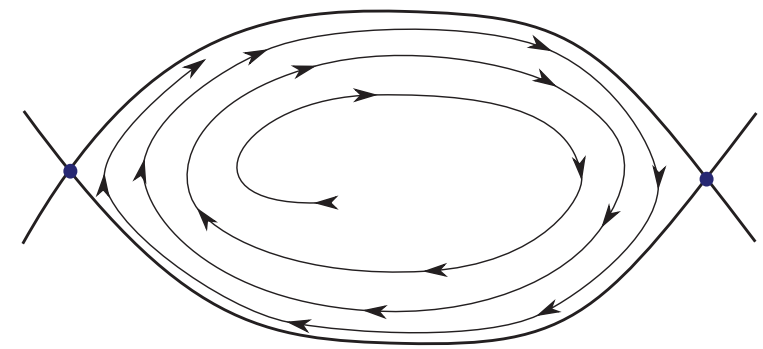

FiguRE 23. Bowen's counterexample 
the singular points, the trajectories are made of a sequence of intervals during which the point is almost at rest, near one of the singular points, separated by short phases of fast transitions. The slow intervals become longer and longer. As a result, the time averages "hesitate" between the two Dirac masses on the singular points. The statistics do not converge to any invariant measure; there is no SRB measure.

This counterexample may seem a bit artificial, and in fact no significantly different counterexample is known. As we will see, it is conjectured that the existence of an SRB measure holds generically among all dynamical systems (see also [84]).

Before providing some significative examples, an important word of caution. Until now we have only considered the topological equivalence between flows: a homeomorphism mapping the orbits of one flow to those of another one. Of course, such a homeomorphism is often singular w.r.t. the Lebesgue measure, so it does not map an SRB measure of the first flow to an SRB measure of the second flow...

The simplest example is given by an expanding map on the circle, which stands as a model for all further developments. It is a map $\psi$, say infinitely differentiable, from the circle to itself, with derivative always greater than 1 . Of course, such a map cannot be bijective. One can show that $\psi$ is topologically conjugate to $x \in \mathbb{R} / \mathbb{Z} \mapsto d . x \in \mathbb{R} / \mathbb{Z}$ for a certain integer $d(d \neq \pm 1)$ but, as we have just noticed, this does not imply any special property regarding the Lebesgue measure.

The Distortion Lemma is a simple, yet fundamental idea. Consider a branch $F_{k}$ of the inverse of $\psi^{k}$, defined on an interval $I$ of the circle. This branch can be written as $f_{k-1} \circ f_{k-2} \circ \cdots \circ f_{0}$, where each $f_{i}$ is one of the inverse branches of $\psi$. To evaluate the difference $\left(\log F_{k}^{\prime}\right)\left(x_{0}\right)-\left(\log F_{k}^{\prime}\right)\left(y_{0}\right)$, let us set $x_{i}=f_{i} \circ \cdots \circ f_{0}\left(x_{0}\right)$ and $y_{i}=f_{i} \circ \cdots \circ f_{0}\left(y_{0}\right)$. We observe that $\left|x_{i}-y_{i}\right| \leqslant \delta^{i}\left|x_{0}-y_{0}\right|$ for some $\delta<1$, which allows to write:

$$
\begin{aligned}
\left|\left(\log F_{k}^{\prime}\right)(x)-\left(\log F_{k}^{\prime}\right)(y)\right| & \leqslant \sum_{i=0}^{k-1}\left|\left(\log f_{i}^{\prime}\right)\left(x_{i}\right)-\left(\log f_{i}^{\prime}\right)\left(y_{i}\right)\right| \\
& \leqslant C \sum_{i=0}^{k-1} \delta^{i}\left|x_{0}-y_{0}\right| \\
& \leqslant \frac{C}{1-\delta}\left|x_{0}-y_{0}\right|
\end{aligned}
$$

where $C$ is an upper bound for the derivative of $\left(\log f_{i}^{\prime}\right)$. This shows that the quotients $F_{k}^{\prime}(x) / F_{k}^{\prime}(y)$ are bounded uniformly w.r.t. $k$ and the chosen branch. This is the statement of the lemma.

Now let Leb be the Lebesgue measure, and Leb $_{k}$ its push-forward through $\psi^{k}$. The density of $\operatorname{Leb}_{k}$ w.r.t. Leb is the sum of the derivatives of all the inverse branches of $\psi^{k}$. This density is therefore bounded from below and from above by two positive constants, independently of the number $k$ of iterations.

It is quite easy to check that any weak limit of the Birkhoff averages $\left(\sum_{1}^{k} \operatorname{Leb}_{i}\right) / k$ is an measure invariant through $\psi$ which is absolutely continuous 
w.r.t. Leb, with density bounded by the same constants. We have thus shown the existence of an invariant measure absolutely continuous w.r.t. the Lebesgue measure.

One can then show that $\psi$ is ergodic w.r.t. the Lebesgue measure: if $A \subset \mathbb{R} / \mathbb{Z}$ is invariant through $\psi$ (meaning that $\psi^{-1}(A)=A$ ), then the Lebesgue measure of $A$ or of its complement vanishes. This property is also a consequence of the Distortion Lemma. Assume the Lebesgue measure of $A$ is nonzero; one can then find intervals $I_{k}$ such that $\operatorname{Leb}\left(I_{k} \cap A\right) / \operatorname{Leb}\left(I_{k}\right)$ goes to 1 when $k \rightarrow \infty$, provided we allow the lengths of the $I_{k}$ to be arbitrary small. If we now set $J_{k}=\psi^{n_{k}}\left(I_{k}\right)$ for some appropriate $n_{k}$, the Distortion Lemma shows that $\operatorname{Leb}\left(J_{k} \cap A\right) / \operatorname{Leb}\left(J_{k}\right)$ also goes to 1 , but this time we may assume that the lengths of the $J_{k}$ are bounded from below. Hence, we can find intervals of lengths bounded from below, which contain an arbitrary large proportion of $A$. In the limit, we obtain a nonempty interval $J_{\infty}$ such that $\operatorname{Leb}\left(J_{\infty} \cap A\right)=\operatorname{Leb}\left(J_{\infty}\right)$, implying that $A$ has a nonempty interior, up to a negligible set. If we expand once more, using the fact that $\psi$ has a dense orbit, we finally show that $A$ has full measure.

We have just shown that there exists a unique invariant measure absolutely continuous w.r.t. the Lebesgue measure, and the Birkhoff ergodic theorem then implies that this measure is SRB.

This proof is quite easy, and it has been generalized in many ways, under less and less hyperbolicity assumptions.

The easiest generalization concerns an expanding map $\psi: V \rightarrow V$, where $V$ may have dimension greater than 1 . One shows analogously that there exists an invariant measure absolutely continuous w.r.t. the Lebesgue measure, and ergodic. For this it suffices to replace the estimates on the derivatives by estimates on Jacobian determinants.

The first really different case is that of a hyperbolic attractor. The situation is as follows: a diffeomorphism $\phi$ preserves a compact set $\Lambda$ and the tangent bundle $T_{\Lambda} V$ above $\Lambda$ splits into the sum $E^{s} \oplus E^{u}$ of a fibre made of uniformly contracted vectors, and another fibre made of uniformly expanded ones. We assume that $\Lambda$ is an attractor, meaning that for each $x \in \Lambda$, the unstable manifold $W^{u}(x)$ - made of the points whose past orbit is asymptotic to that of $x$ - is fully contained in $\Lambda$. Each point $y$ in an open neighborhood $U$ of $\Lambda$ then lies on the stable manifold of a certain point $x \in \Lambda$ : the future orbit of $y$ is asymptotic to that of $x$, and thus accumulates in $\Lambda$.

The set $\Lambda$ is foliated by unstable manifolds, which are uniformly expanded through $\phi$. The idea is then to copy the Distortion Lemma, but only along the expanding directions. One starts from a probability measure supported in a small ball contained in some unstable manifold, and which is absolutely continuous w.r.t. the Lebesgue measure on the unstable manifold. One then successively pushes forward this measure through the diffeomorphism, and tries to show a weak convergence by time averaging the obtained sequence of measures. We don't need to estimate the full Jacobian determinants, but rather the unstable Jacobians, computed along $E^{u}$. 
The result is as follows: one obtains an invariant measure which is not necessarily absolutely continuous w.r.t. the Lebesgue measure, but which is absolutely continuous when restricted on the unstable manifolds. The last statement needs some explanations. Locally, near a point $x \in \Lambda$, the situation is that of a product $B^{u} \times B^{s}$ of an open ball in the unstable manifold $W^{u}(x)$ and an open ball in the stable manifold $W^{s}(x)$. Two points with the same first coordinate (resp. the same second coordinate) lie in the same stable manifold (resp. unstable manifold). A measure $\mu$ can then be "disintegrated" in these local coordinates: if $A \subset B^{u} \times B^{s}$,

$$
\mu(A)=\int_{T} \mu_{t}\left(A \cap\left(B^{u} \times\{t\}\right)\right) d \nu(t),
$$

where $\nu$ is a measure on $B^{s}$ and $\mu_{t}$ is a measure on $B^{u}$ defined for $\nu$-almost all $t \in B^{s}$. The measures constructed above by time averaging are such that $\mu_{t}$ is absolutely continuous w.r.t. the Lebesgue measure on the unstable leaves, for $\nu$-almost all $t$. Technically, $\mu$ is called an (unstable) Gibbs state.

There remains to show that these Gibbs states are SRB measures. If we consider the basin $B(\mu)$, namely the set of points for which, for any continuous test function, the time average is equal to the $\mu$-average, this basin is clearly a union of stable manifolds: if two points have asymptotic future orbits, their time averages coincide. Besides, the defining property of Gibbs states implies that a set of full $\mu$-measure intersects almost all the unstable manifolds along parts of full Lebesgue measure. Does this indeed imply that the basin has positive Lebesgue measure in $V$ ? To show it, one needs to establish that a subset of $B^{u} \times B^{s}$ which is a union of vertical balls, and meets each horizontal ball on a set of full Lebesgue measure in $B^{u}$, has positive full Lebesgue measure in $B^{u} \times B^{s}$. This would be the case if the parametrization of a neighborhood of $x$ through $B^{u} \times B^{u}$ were differentiable, unfortunately it is generally not the case. On the other hand, one fundamental technical result states that the "holonomies" of these stable and unstable foliations are absolutely continuous w.r.t. the Lebesgue measure: this means that, in the coordinates $B^{u} \times B^{s}$, the projection on the first coordinate allows to identify different unstable balls, and these identifications all preserve the sets of zero Lebesgue measure. One can then apply Fubini's theorem to conclude that a Gibbs state is indeed an SRB measure: its basin $B(\mu)$ has full Lebesgue measure in the open neighborhood $U$ of $\Lambda$. These results make up most of $[71,66,17]$.

To go further, one may relax the hyperbolicity assumptions. For instance, one may assume that the diffeomorphism $\phi$ is partially hyperbolic: it admits an invariant decomposition $E^{u} \oplus E$. The vectors in $E^{u}$ are still uniformly expanded, but we don't assume any more that the vectors in $E$ are contracted. On the other hand, if a vector in $E$ is expanded, it should be so less strongly than the vectors in $E^{u}$ : one speaks of a dominated decomposition. Under this hypothesis, it is not difficult to copy the previous reasoning and show the existence of unstable Gibbs states. On the other hand, this hypothesis is not sufficient to show that the Gibbs states are SRB measures: the condition ensuring the existence of sufficiently many stable manifolds, such that almost every point of the open $U$ is asymptotic to a 
point in the attractor, is lacking. To show that a Gibbs state $\mu$ is an SRB measure, it is enough for instance to require that for $\mu$-almost every point $x \in \Lambda$ the vectors in $E_{x}$ are exponentially contracted.

The quest for the weakest conditions allowing to show the existence of SRB measures is summarized in Chapter 11 of the book [16]. This question is fundamental since, as we will see, one hopes that "almost all" diffeomorphisms admit SRB measures.

\subsection{Ergodic theory of the Lorenz attractor}

The geometric Lorenz models are not hyperbolic, hence are not encompassed by the works of Sinai, Ruelle and Bowen we have just summarized. On the other hand, the Lorenz flow is singular hyperbolic. At each point $x$ of the attractor $\Lambda$, the tangent space can be split into a direct sum of a line $E_{x}^{s}$ and a plane $F_{x}$, such that the vectors of $E^{s}$ are uniformly contracted, while a vector in $F_{x}$ is "less contracted" than the vectors in $E^{s}$. Of course the plane $F_{x}$ contains the Lorenz vector field. Notice that a vector field is invariant through the flow it generates, so at each nonsingular point it provides a nonexpanding direction. One expects to find expansion in $F_{x}$, in the direction transverse to the field, but this expansion may not be uniform, due to the defect of hyperbolicity. For each point $x \in \Lambda$ we have a stable manifold $W^{s}(x)$, which is a curve "transverse" to the attractor.

We have also studied the structure of the first return map on the square $C$ : it has all the characteristics of a hyperbolic map. This allows, e.g., to show the existence of an invariant measure absolutely continuous w.r.t. the Lebesgue measure for the map $f$ on the interval. The return map of an orbit on the square diverges (logarithmically) when going to the discontinuity of $f$. This poses technical difficulties (but, quite strangely, sometimes also simplifies the work). Most of these technical difficulties have been overcome, and we now understand quite well the ergodic behavior of the Lorenz flows.

One of the first results, obtained in the 1980s, is that the Lorenz attractor supports a unique SRB measure $[21,56]$.

Moreover, the unstable Lyapunov exponent for this measure $\mu$ is positive. This means that for $\mu$-almost every point $x \in \Lambda$ one may find a line $E_{x}^{u}$ contained in the plane $F_{x}$, such that for $v \in E_{x}^{u}$,

$$
\lim _{T \rightarrow \infty} \frac{1}{T} \log \left\|d \phi^{T}(v)\right\|=\lambda>0,
$$

with $\lambda$ independent of $x$. In other words, the dynamics exponentially expands almost all the vectors. Of course, this expansion is not uniform.

One can then apply Pesin's theory for the Lyapunov exponents, which allows to construct unstable manifolds. Precisely, for $\mu$-almost all point $x \in \Lambda$, the set of points $y$ with past orbits asymptotic to that of $x$ is a smooth curve $W_{x}^{u}$ contained in $\Lambda$. In a way, $\mu$ is hyperbolic almost everywhere, with a splitting of the tangent space into three directions: $E_{x}^{s}$ (which continuously depends on $x$ ), $E_{x}^{u}$ (defined 
almost everywhere) and the direction of the vector field (defined everywhere except on the singular point).

The fact that this SRB measure is mixing has been shown only recently [46]: if $A, B$ are two measurable subsets of $U$, one has

$$
\lim _{t \rightarrow+\infty} \mu\left(\phi^{t}(A) \cap B\right)=\mu(A) \mu(B) .
$$

The most complete result is that obtained recently by Holland and Melbourne [37]. Consider a smooth function $u$ defined on the open set $U$, and to simplify the statement, assume that its $\mu$-average vanishes. Then, there exists a Brownian motion $W_{T}$ of variance $\sigma^{2} \geqslant 0$ and $\epsilon>0$ such that, $\mu$-almost surely,

$$
\int_{0}^{T} u \circ \phi^{t} d t=W_{T}+O\left(T^{\frac{1}{2}-\varepsilon}\right) \quad \text { when } \quad t \rightarrow+\infty .
$$

That is, the Birkhoff sums oscillate like a Brownian motion around their average value. This implies a Central Limit Theorem and a Law of the iterated logarithm.

Let us also mention the preprint [24] which analyzes in detail the statistics of the recurrence times in a small ball.

\section{The butterfly within the global picture}

\subsection{Singular hyperbolicity}

The Lorenz attractor is mainly an example featuring a certain number of properties:

- it is complicated and chaotic, yet not hyperbolic;

- its dynamics can nevertheless be described reasonably well, both from the topological and ergodic points of view;

- it admits "some" hyperbolicity;

- it is robust: any perturbation of a Lorenz model is another Lorenz model.

The central question is to determine whether it constitutes a "significative" example. The aim is to find sufficiently general qualitative properties on a dynamics, allowing to show that it resembles a Lorenz attractor.

Before stating some recent results in this direction, I shall first explain a precursory theorem of Mañé, dating back to 1982 [47]. Let $\phi$ be a diffeomorphism on a compact manifold $V$, and $\Lambda \subset V$ a compact invariant set. $\Lambda$ is said to be transitive if it contains a dense orbit (w.r.t. the induced topology in $\Lambda$ ). It is said to be maximal if there exists an open neighborhood $U$ such that for each point $x$ outside $\Lambda$, the orbit of $x$ will leave $U$ in the past or in the future. Finally, $\Lambda$ is said to be robustly transitive if these properties persist under perturbation: there exists a neighborhood $\mathcal{V}$ of $\phi$ in the space of $C^{1}$ diffeomorphisms, such that if $\psi \in \mathcal{V}$, the compact set $\Lambda_{\psi}=\cap_{k \in \mathbb{Z}} \psi^{k}(U)$ is nonempty and transitive. Mañé's theorem is the first one showing that hyperbolicity can result from dynamical conditions of this kind. It states that in dimension 2, the robustly transitive sets are exactly the hyperbolic sets. Such a result cannot be generalized to higher dimension, where 
one should try to obtain some partial hyperbolicity. Chapter 7 in [16] is entirely devoted to describing the recent results in this direction.

In the case of vector fields in dimension 3, the main result has been obtained by Morales, Pacifico and Pujals in 2004 [49]. Let $\phi^{t}$ be a flow on a manifold $V$, preserving a compact set $\Lambda$. Let us recall a definition given in Section 5.2: $\Lambda$ is said to be singular hyperbolic if - like in the case of the Lorenz model - the tangent space $T_{\Lambda} V$ decomposes into $E^{s} \oplus F$, such that:

- $E_{x}^{s}$ and $F_{x}$ depend continuously on the point $x \in \Lambda$ and are invariant through the differential of $\phi^{t}$;

- the vectors in $E_{x}^{s}$ are uniformly contracted by the flow;

- the decomposition $E_{x}^{s} \oplus F_{x}$ is dominated: if $u \in F_{x}$ and $v \in E_{x}^{s}$ are unitary, one has $\left\|d \phi^{t}(u)\right\| \geqslant C \exp (\lambda t)\left\|d \phi^{t}(v)\right\|$ for all $t>0$;

- the flow expands the bidimensional volume on $F_{x}: \operatorname{det}\left(d \phi^{t} \mid F_{x}\right) \geqslant C \exp (\lambda t)$ for all $t>0$.

The main theorem in [49] states that if a compact invariant set $\Lambda$ of a three-dimensional flow is robustly transitive, then:

- $\Lambda$ is singular hyperbolic;

- if $\Lambda$ does not contain any singular point, it is a hyperbolic set;

- all the field singularities contained in $\Lambda$ are of Lorenz type; the three eigenvalues $a_{1}<a_{2}<a_{3}$ of the linearization satisfy $a_{1}<0<a_{3}$ and either $-a_{3}<a_{2}<0$ (index 2$)$ or $0<a_{2}<-a_{1}($ index 1$)$;

- all the singularities have the same index and $\Lambda$ is an attractor or a repeller, (i.e., an attractor for the reversed field) depending on the value 2, resp. 1, of the index.

Hence, this theorem provides the Lorenz attractors (more precisely, the dynamics of the same family) with a new status: they are singular hyperbolic attractors. They represent, in dimension 3, a qualitative phenomenon: robust transitivity. One can read a sketch of proof, as well as some complements, in Chapter 9 of [16].

One still needs to generalize the results available for the Lorenz attractor to all singular hyperbolic attractors. The topological understanding of these objects is still incomplete, although we know, for instance, that periodic orbits are dense in the attractor [9]. The ergodic understanding is now much more advanced, and rather close to the case of the Lorenz attractors; for instance, there exists an SRB measure, the basin of which contains almost all the points of an open neighborhood of the attractor [6].

\subsection{Palis's big picture}

The history of dynamical systems seems to be punctuated by a long sequence of hopes, soon to be abandoned ... A Morse-Smale world, replaced by a world of hyperbolic attractors, in turn destroyed by an abundance of examples like Lorenz's model. Yet, dynamicists do not lack optimism, and they do not hesitate to brush a world view according to their present belief, (naively) hoping that their view will not soon be obsolete. Palis has formulated such a vision in three articles in 1995, 
2005 and 2008 [51, 52, 53]. He states there an ensemble of conjectures describing the dynamics of "almost all" diffeomorphisms or flows. These conjectures are necessarily technical, and it is not useful to describe them in detail here. I will only give a sketch of the general spirit.

The first difficulty - which is not specific to this domain - is to give a meaning to "almost all" dynamics. The initial idea from the 1960s was to describe an open dense set in the space of dynamical systems, or at least, a countable intersection of open dense sets, in order to use Baire genericity. Yet, this notion has proved too strict. It is well known that no notion of "Lebesgue measure" can be defined on a space of infinite dimension, but a substitute - prevalence - seems to impose itself little by little. Let $\mathcal{P}$ be a subset of the set $\operatorname{Diff}(V)$ of diffeomorphisms (of a certain regularity) on a manifold $V$. We then consider a family of diffeomorphisms $\phi_{\nu}$ on $V$ depending on a parameter $\nu$, which for instance takes values in a ball in $\mathbb{R}^{p}$. One can then evaluate the Lebesgue measure of the set of $\nu$ such that $\phi_{\nu}$ belongs to $\mathcal{P}$. Of course, if the family $\phi_{\nu}$ is degenerate, for instance if it does not depend on $\nu$, it can completely avoid $\mathcal{P}$. The set $\mathcal{P}$ is said to be prevalent if, for a generic family $\phi_{\nu}$ (in the Baire sense within the space of families), the set of $\nu$ such that $\phi_{\nu}$ belongs to $\mathcal{P}$ has full Lebesgue measure (within the ball covered by the parameter $\nu$ ).

Palis's finiteness conjecture reads as follows:

The following properties are prevalent among the diffeomorphisms or the flows on a given compact manifold:

- there exist finitely many attractors $\Lambda_{1}, \ldots, \Lambda_{k}$;

- each attractor admits an SRB measure;

- the union of the basins of attraction of the SRB measures covers almost all the manifold, in the sense of the Lebesgue measure.

As for the lost hope of generic structural stability, it has been replaced by stochastic stability. Consider a family of diffeomorphisms $\phi_{\nu}$ depending on a parameter $\nu$ in a neighborhood of the origin in $\mathbb{R}^{p}$, with $\phi_{0}=\phi$. Choose a point $x_{0}$ in an attractor $\Lambda$, a small $\varepsilon>0$, and consider the random sequence of points

$$
x_{0} \quad ; \quad x_{1}=\phi_{\nu_{1}}\left(x_{0}\right) ; x_{2}=\phi_{\nu_{2}}\left(x_{1}\right) ; x_{k}=\phi_{\nu_{k}}\left(x_{k-1}\right) ; \ldots,
$$

where the parameters $\nu_{i}$ are randomly chosen in the ball of radius $\varepsilon$. The SRB measure $\mu$ on the attractor $\Lambda$ is said to be stochastically stable if, for any neighborhood $\mathcal{U}$ of $\mu$ in the weak topology on measures, and for any family $\phi_{\nu}$, one can find $\varepsilon$ such that, for almost all choices of the above random sequence, the average over the Dirac masses

$$
\frac{1}{k+1}\left(\delta_{x_{0}}+\delta_{x_{1}}+\cdots+\delta_{x_{k}}\right)
$$

has a limit when $k \rightarrow \infty$, and the limit belongs to $\mathcal{U}$. In other words, through a stochastic perturbation, the statistical behavior "does not change too much"...

A second part of Palis's conjectures states that stochastic stability is prevalent. 

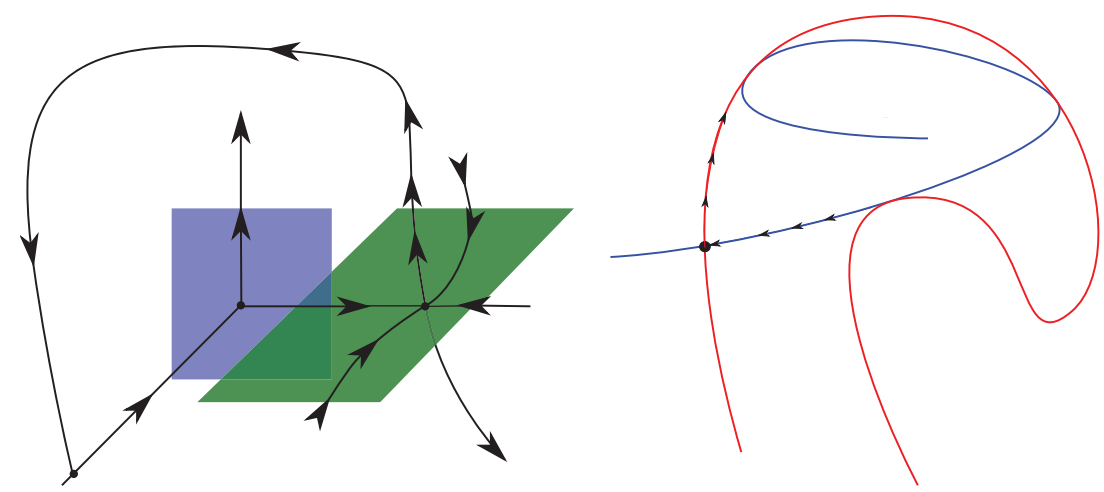

FIGURE 24. A heteroclinic orbit and a homoclinic tangency

These conjectures come with a general strategy of proof. The idea is to explain the non-density of hyperbolicity through the appearance of a few paradigmatic phenomena, one of which is the Lorenz type attractor.

So far only a few nonhyperbolic phenomena have been identified:

- heterodimensional cycles

Such a cycle consists in two periodic hyperbolic points $x, y$ in the same transitive set, the unstable manifolds of which have different dimensions, and such that the stable manifold of each point transversally intersects the unstable manifold of the other. It is, of course, an obstruction to hyperbolicity. For diffeomorphisms, this type of cycle only appears for dimensions $\geq 3$.

- homoclinic tangencies

A homoclinic tangency is a nontransverse intersection between the stable and unstable manifolds of a same periodic point. Once more, it is an obstruction to hyperbolicity.

- singular cycles

In the case of flows, the possible presence of singularities offers new possibilities. A cycle is given by a finite sequence $\gamma_{1}, \ldots, \gamma_{l}$ of hyperbolic singularities or periodic orbits, which are cyclically connected by regular orbits: there are points $x_{1}, \ldots, x_{l}$ such that the $\alpha$-limit set of $x_{i}$ is $\gamma_{i}$ and the $\omega$-limit set of $x_{i}$ is $\gamma_{i+1}$ (where $\gamma_{l+1}=\gamma_{1}$ ). The cycle is singular provided at least one of the $\gamma_{i}$ is a singularity. These singular cycles appear densely in the family of Lorenz models: the two unstable curves starting from the singular point spiral in the attractor, and can be localized in the stable manifold of this singular point. This happens if the point 0 is periodic for the map $f$ on $[-1 / 2,1 / 2]$ we have studied.

Palis proposes that on a given compact manifold, the set of vectors fields which are either hyperbolic or display one of these phenomena is dense (in the $C^{r}$ topology). 
In dimension 3, he conjectures that the union of the vector fields which are either hyperbolic, or admit a homoclinic tangency, or have attractors (or repellers) of Lorenz type, is dense (in the $C^{r}$ topology).

Arroyo and Rodriguez-Hertz have made progresses in this direction, by showing that a three-dimensional field can be approached, in the $C^{1}$ topology, by fields which are either hyperbolic, or present a homoclinic tangency or a singular cycle $[10]$.

In any case, the Lorenz attractor displays phenomena which could be characteristic of "typical chaos". At least in the framework of mathematical chaos, since the relevance of the Lorenz model to describe meteorological phenomena remains widely open [64].

\subsection{A long and beautiful sentence}

To finish, here is a quotation by Buffon dating back to 1783, and showing that his view of an ergodic and mixing world was quite close from Lorenz's one [20].

[...] tout s'opère, parce qu'à force de temps tout se rencontre, et que dans la libre étendue des espaces et dans la succession continue du mouvement, toute matière est remuée, toute forme donnée, toute figure imprimée; ainsi tout se rapproche ou s'éloigne, tout s'unit ou se fuit, tout se combine ou s'oppose, tout se produit ou se détruit par des forces relatives ou contraires, qui seules sont constantes, et se balançant sans se nuire, animent l'Univers et en font un théâtre de scènes toujours nouvelles, et d'objets sans cesse renaissants.

\section{References}

[1] R. Abraham and S. Smale, Nongenericity of $\Omega$-stability. Global Analysis (Proc. Sympos. Pure Math., Vol. XIV, Berkeley, Calif., 1968) pp. 5-8 Amer. Math. Soc., Providence, R.I. 1970.

[2] V.S. Afraimovich, V.V. Bykov and L.P. Shil'nikov, The origin and structure of the Lorenz attractor. (En russe), Dokl. Akad. Nauk SSSR 234 no. 2, 336-339 (1977).

[3] D.V. Anosov, Roughness of geodesic flows on compact Riemannian manifolds of negative curvature. (En russe), Dokl. Akad. Nauk SSSR 145, 707-709 (1962).

[4] D.V. Anosov, Dynamical systems in the 1960s: the hyperbolic revolution. Mathematical events of the twentieth century, 1-17, Springer, Berlin, 2006.

[5] A. Andronov. and L. Pontrjagin, Systèmes grossiers. Dokl. Akad. Nauk SSSR 14 (1937).

[6] V. Araújo, E.R. Pujals, M.J. Pacifico and M. Viana, Singular-hyperbolic attractors are chaotic. Transactions of the A.M.S. 361, 2431-2485 (2009).

[7] V. Arnold, Sur la géométrie différentielle des groupes de Lie de dimension infinie et ses applications à l'hydrodynamique des fluides parfaits. Annales de l'institut Fourier 16 no. 1, 319-361 (1966). 
[8] D. Aubin and A. Dahan Dalmedico, Writing the history of dynamical systems and chaos: longue durée and revolution, disciplines and cultures. Historia Math. 29 no. 3, 273-339 (2002).

[9] A. Arroyo and E. Pujals, Dynamical properties of singular-hyperbolic attractors. Discrete Contin. Dyn. Syst. 19 no. 1, 67-87 (2007).

[10] A. Arroyo and F. Rodriguez Hertz, Homoclinic bifurcations and uniform hyperbolicity for three-dimensional flows. Ann. Inst. H. Poincaré Anal. Non Linéaire 20 no. 5, 805-841 (2003).

[11] J. Barrow-Green, Poincaré and the three body problem. History of Mathematics, 11. American Mathematical Society, Providence, RI; London Mathematical Society, London, 1997. xvi+272 pp.

[12] F. Béguin, Le mémoire de Poincaré pour le prix du roi Oscar. In L'héritage scientifique de Poincaré, éd. Charpentier, Ghys, Lesne, éditions Belin 2006.

[13] G.D. Birkhoff, Nouvelles recherches sur les systèmes dynamiques. Mem. Pont. Acad. Sci. Nov. Lyncaei 53, 85-216 (1935).

[14] J. Birman and I. Kofman, A new twist on Lorenz links. J. Topol. 2 no. 2, 227-248 (2009).

[15] J. Birman and R.F. Williams, Knotted periodic orbits in dynamical systems, I. Lorenz's equations. Topology 22 no. 1, 47-82 (1983).

[16] C. Bonatti, L. Díaz and M. Viana, Dynamics beyond uniform hyperbolicity. A global geometric and probabilistic perspective. Encyclopaedia of Mathematical Sciences, 102. Mathematical Physics, III. Springer-Verlag, Berlin, 2005.

[17] R. Bowen, Equilibrium states and the ergodic theory of Anosov diffeomorphisms. Lecture Notes in Mathematics 470, Springer-Verlag, Berlin, 1975.

[18] R. Bowen, On Axiom A diffeomorphisms. Regional Conference Series in Mathematics, No. 35. American Mathematical Society, Providence, R.I., 1978. vii+45 pp.

[19] R. Bowen and D. Ruelle, The ergodic theory of Axiom A flows. Invent. Math. 29 no. 3, 181-202 (1975).

[20] G.L. Buffon, Du Soufre. Histoire Naturelle des minéraux, tome 2, 1783.

[21] L.A. Bunimovich, Statistical properties of Lorenz attractors. Nonlinear dynamics and turbulence, Pitman, p. 7192, 1983.

[22] P. Dehornoy, Les nœuds de Lorenz. to be published in L'Enseignement mathématique, arXiv:0904.2437

[23] P. Duhem, La théorie physique; son objet, sa structure. 1906. English transl. by P.P. Wiener, The aim and structure of physical theory, Princeton University Press, 1954.

[24] S. Galatolo and M.J. Pacifico, Lorenz-like flows: exponential decay of correlations for the Poincaré map, logarithm law, quantitative recurrence. Ergod. Th. Dynam. Sys. 30, 1703-1737 (2010).

[25] R. Ghrist, Branched two-manifolds supporting all links. Topology 36 no. 2, 423-448 (1997).

[26] E. Ghys, Knots and dynamics. International Congress of Mathematicians. Vol. I, pp. 247-277, Eur. Math. Soc., Zürich, 2007.

[27] E. Ghys, Right-handed vector fields \& the Lorenz attractor. Jpn. J. Math 4 no. 1, 47-61 (2009). 
[28] E. Ghys and J. Leys, Lorenz and modular flows: a visual introduction. American Math. Soc. Feature Column, November 2006, www . ams .org/featurecolumn/archive/lorenz.html

[29] J. Gleick, Chaos: Making a New Science. Viking Penguin, 1987.

[30] M. Gromov, Three remarks on geodesic dynamics and fundamental group. Enseign. Math. 46 no. 3-4, 391-402 (2002).

[31] J. Guckenheimer, A strange, strange attractor. In The Hopf Bifurcation, Marsden and McCracken, eds. Appl. Math. Sci., Springer-Verlag, (1976).

[32] J. Guckenheimer, The Lorenz Equations: Bifurcations, Chaos, and Strange Attractors. Amer. Math. Monthly 91 no. 5, 325-326 (1984).

[33] J. Guckenheimer and R.F. Williams, Structural stability of Lorenz attractors. Inst. Hautes études Sci. Publ. Math. 50, 59-72 (1979).

[34] J. Hadamard, Les surfaces à courbures opposées et leurs lignes géodésiques. Journal de mathématiques pures et appliquées 5e série 4, 27-74 (1898).

[35] J.T. Halbert and J.A. Yorke, Modeling a chaotic machine's dynamics as a linear map on a "square sphere" . http://www.math.umd.edu/ halbert/taffy-paper-1.pdf

[36] B. Hasselblatt, Hyperbolic dynamical systems. Handbook of dynamical systems, Vol. 1A, pp. 239-319, North-Holland, Amsterdam, 2002.

[37] M. Holland and I. Melbourne, Central limit theorems and invariance principles for Lorenz attractors. J. Lond. Math. Soc. (2) 76 no. 2, 345-364 (2007).

[38] J.-P. Kahane, Hasard et déterminisme chez Laplace. Les Cahiers Rationalistes 593 - mars-avril 2008.

[39] S. Kuznetsov, Plykin-type attractor in nonautonomous coupled oscillators. Chaos 19, 013114 (2009).

[40] O. Lanford, An introduction to the Lorenz system. Papers from the Duke Turbulence Conference (Duke Univ., Durham, N.C., 1976), Paper No. 4, i+21 pp. Duke Univ. Math. Ser., Vol. III, Duke Univ., Durham, N.C., 1977.

[41] P.S. Laplace, Essai philosophique sur les probabilités. 1814. English transl. by A.I. Dale, Philosophical essay on probabilities, Springer, 1995

[42] E.N. Lorenz, Maximum simplification of the dynamic equations. Tellus 12, 243-254 (1960).

[43] E.N. Lorenz, The statistical prediction of solutions of dynamic equations. Proc. Internat. Sympos. Numerical Weather Prediction, Tokyo, pp. 629-635.

[44] E.N. Lorenz, Deterministic non periodic flow. J. Atmosph. Sci. 20, 130-141 (1963).

[45] E.N. Lorenz, Predictability: does the flap of a butterfly's wings in Brazil set off a tornado in Texas? 139th Annual Meeting of the American Association for the Advancement of Science (29 Dec. 1972), in Essence of Chaos (1995), Appendix 1, 181.

[46] S. Luzzatto, I. Melbourne and F. Paccaut, The Lorenz attractor is mixing. Comm. Math. Phys. 260 no. 2, 393-401 (2005).

[47] R. Mañé, An ergodic closing lemma. Ann. of Math. 116 no. 3, 503-540 (1982).

[48] J.C. Maxwell, Matter and Motion. 1876, rééd. Dover (1952).

[49] C.A. Morales, M.J. Pacifico and E.R. Pujals, Robust transitive singular sets for 3flows are partially hyperbolic attractors or repellers. Ann. of Math. 160 no. 2, 375-432 (2004). 
[50] H.M. Morse, A one-to-one representation of geodesics on a surface of negative curvature. Amer. J. Math. 43 no. 1, 33-51 (1921).

[51] J. Palis, A global view of dynamics and a conjecture on the denseness of finitude of attractors. Géométrie complexe et systèmes dynamiques (Orsay, 1995), Astérisque No. 261 (2000), xiii-xiv, pp. 335-347.

[52] J. Palis, A global perspective for non-conservative dynamics. Ann. Inst. H. Poincaré Anal. Non Linéaire 22 no. 4, 485-507 (2005).

[53] J. Palis, Open questions leading to a global perspective in dynamics. Nonlinearity 21 no. 4, T37-T43 (2008).

[54] J. Palis and F. Takens, Hyperbolicity and sensitive chaotic dynamics at homoclinic bifurcations. Cambridge Studies in Advanced Mathematics, 35. Cambridge University Press, Cambridge, 1993. x+234 pp.

[55] M. Peixoto, Structural stability on two-dimensional manifolds. Topology 1, 101-120 (1962).

[56] Ya.B. Pesin, Dynamical systems with generalized hyperbolic attractors: hyperbolic, ergodic and topological properties. Ergodic Theory Dynam. Systems 12 no. 1, 123151 (1992).

[57] H. Poincaré, Mémoire sur les courbes définies par une équation différentielle. Journal de mathématiques pures et appliquées 7, 375-422 (1881).

[58] H. Poincaré, Sur le problème des trois corps et les équations de la dynamique. Acta Mathematica 13, 1-270 (1890).

[59] H. Poincaré, Les méthodes nouvelles de la mécanique céleste. Gauthier-Villars, Paris, vol. 3, (1892-1899). English transl. New methods of celestial mechanics, D.L. Goroff (ed.), American Institute of Physics, 1993

[60] H. Poincaré, Sur les lignes géodésiques des surfaces convexes. Trans. Amer. Math. Soc. 6, 237-274 (1905).

[61] H. Poincaré, Le hasard. Revue du Mois 3, 257-276 (1907).

[62] H. Poincaré, Science et méthode. Flammarion, (1908). English transl. by F. Maitland, Science and Method, T. Nelson and Sons, London, 1914.

[63] A. Robadey, Différentes modalités de travail sur le général dans les recherches de Poincaré sur les systèmes dynamiques. Thèse, Paris 7, 2005.

[64] R. Robert, L'effet papillon n'existe plus! Gaz. Math. 90, 11-25 (2001).

[65] D. Ruelle, The Lorenz attractor and the problem of turbulence. Turbulence and Navier-Stokes equations (Proc. Conf., Univ. Paris-Sud, Orsay, 1975), pp. 146-158, Lecture Notes in Math., 565, Springer, Berlin, (1976).

[66] D. Ruelle, A measure associated with axiom-A attractors, Amer. J. Math. 98 no. 3, 619-654 (1976).

[67] D. Ruelle and F. Takens, On the nature of turbulence. Commun. Math. Phys. 20, 167-192 (1971).

[68] L.P. Shil'nikov, Homoclinic trajectories: from Poincaré to the present. Mathematical events of the twentieth century, 347-370, Springer, Berlin, 2006.

[69] M. Shub, What is ... a horseshoe?, Notices Amer. Math. Soc. 52 no. 5, 516-517 (2005).

[70] M. Shub, et S. Smale, Beyond hyperbolicity. Ann. of Math. (2) 96, 587-591(1972). 
[71] Ja.G. Sinai, Gibbs measures in ergodic theory. (En russe), Uspehi Mat. Nauk 27, no. 4(166), 21-64 (1972).

[72] S. Smale, On dynamical systems. Bol. Soc. Mat. Mexicana 5, 195-198 (1960).

[73] S. Smale, A structurally stable differentiable homeomorphism with an infinite number of periodic points. Qualitative methods in the theory of non-linear vibrations (Proc. Internat. Sympos. Non-linear Vibrations, Vol. II, 1961) pp. 365-366 Izdat. Akad. Nauk Ukrain. SSR, Kiev 1963.

[74] S. Smale, Diffeomorphisms with many periodic points. Differential and Combinatorial Topology (A Symposium in Honor of Marston Morse) pp. 63-80, Princeton Univ. Press, Princeton, N.J. 1965.

[75] S. Smale, Structurally stable systems are not dense. Amer. J. Math. 88, 491-496 (1966).

[76] S. Smale, Differentiable dynamical systems. Bull. Amer. Math. Soc. 73, 747-817 (1967).

[77] S. Smale, Finding a horseshoe on the beaches of Rio. Math. Intelligencer 20 no. 1 , 39-44 (1998).

[78] S. Smale, Mathematical problems for the next century. Math. Intelligencer 20 no. 2, 7-15 (1998).

[79] C. Sparrow, The Lorenz equations: bifurcations, chaos, and strange attractors. Applied Mathematical Sciences, 41. Springer-Verlag, New York-Berlin, (1982).

[80] W. Tucker, A rigorous ODE solver and Smale's 14th problem. Found. Comput. Math. 2\$, no. 1, 53-117 (2002).

[81] M. Viana, What's new on Lorenz strange attractors? Math. Intelligencer 22 no. 3, 6-19 (2000).

[82] R.F. Williams, The structure of Lorenz attractors. Inst. Hautes Études Sci. Publ. Math. 50, 73-99 (1979).

[83] R.F. Williams, Lorenz knots are prime. Ergodic Theory Dynam. Systems 4 no. 1, 147-163 (1984).

[84] L.S. Young, What are SRB measures, and which dynamical systems have them? . Dedicated to David Ruelle and Yasha Sinai on the occasion of their 65th birthdays. J. Statist. Phys. 108 no. 5-6, 733-754 (2002).

Étienne Ghys

CNRS - ÉNS Lyon

UMPA, 46 Allée d'Italie

F-69364 Lyon, France 